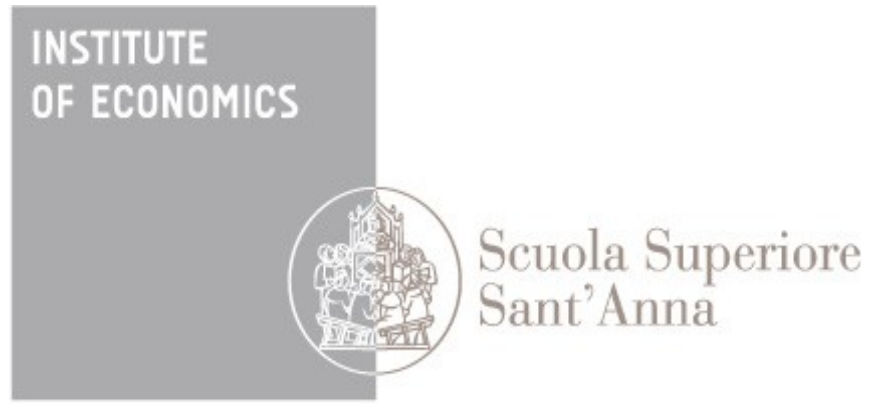

LEM | Laboratory of Economics and Management

Institute of Economics

Scuola Superiore Sant'Anna

Piazza Martiri della Libertà, 33 - 56127 Pisa, Italy ph. +3905088.33 .43$

institute.economics@sssup.it

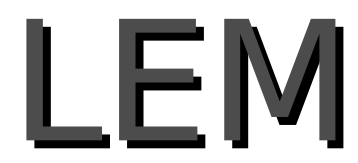

Working Paper Series

\title{
The Anatomy of Government Bond Yields Synchronization in the Eurozone
}

\author{
Claudio Barbieri a,b \\ Mattia Guerini c,a,b \\ Mauro Napoletano a,d,e,b
}

a GREDEG, CNRS, Université Côte d'Azur, France.

${ }^{b}$ Institute of Economics, Scuola Superiore Sant'Anna, Pisa, Italy.

' University of Brescia, Italy.

d Sciences Po, OFCE, France.

e SKEMA Business School, France. 


\title{
The Anatomy of Government Bond Yields Synchronization in the Eurozone
}

\author{
Claudio Barbieri ${ }^{\mathrm{a}, \mathrm{b}} \quad$ Mattia Guerini ${ }^{\mathrm{c}, \mathrm{a}, \mathrm{b}} \quad$ Mauro Napoletano $\mathrm{N}^{\mathrm{a}, \mathrm{d}, \mathrm{e}, \mathrm{b}}$

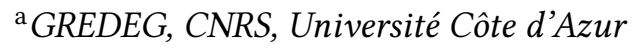 \\ b Institute of Economics, Scuola Superiore "Sant'Anna" \\ ${ }^{\mathrm{c}}$ University of Brescia \\ ${ }^{\mathrm{d}}$ Sciences Po, OFCE \\ ${ }^{\mathrm{e}}$ SKEMA Business School
}

March 3, 2021

\begin{abstract}
We investigate the synchronization of Eurozone's government bond yields at different maturities. For this purpose, we combine principal component analysis with random matrix theory. We find that synchronization depends upon yields maturity. Short-term yields are not synchronized. Medium- and long-term yields, instead, were highly synchronized early after the introduction of the Euro. Synchronization then decreased significantly during the Great Recession and the European Debt Crisis, to partially recover after 2015. We show the existence of a duality between our empirical results and portfolio theory and we point to divergence trades and flight-to-quality effects as a source of the self-sustained yield asynchronous dynamics. Our results envisage synchronization as a requirement for the smooth transmission of conventional monetary policy in the Eurozone.
\end{abstract}

Keywords: Synchronization, Bond Yields, Factor Models, Random Matrix Theory, Monetary policy.

JEL Codes: C38, E43, E58.

$\bowtie$ Claudio Barbieri - claudio.barbieri@univ-cotedazur.fr.

$凶$ Mattia Guerini - mattia.guerini@unibs.it

$\bowtie$ Mauro Napoletano (Corresponding Author)- mauro.napoletano@univ-cotedazur.fr 


\section{Introduction}

Since the introduction of the common currency, the Eurozone has been characterized by a common monetary policy authority and distinct fiscal policy authorities. Hence, government bonds yields are allowed to differ to reflect country-specific characteristics and financial traders can create portfolios comprising the different bonds. In this context, idiosyncratic shocks affect the composition of optimal portfolios and, as a consequence, bond yields. This might give rise to asynchronous yield movements, hindering the transmission mechanism of conventional monetary policy within the union. The Great Recession and the subsequent European Debt Crisis have highlighted this possibility, even endangering the stability of the whole European architecture. A precise assessment of yields' synchronization in the eurozone is therefore crucial for identifying periods in which conventional monetary policies are ineffective and unconventional ones required.

In this paper, we empirically investigate synchronization in government bonds' yields by employing a rich dataset retrieved from the Bloomberg platform at daily frequency, covering the period 2003-2019, for all eurozone economies and for eleven different bond's maturities. We apply a novel procedure based upon Random Matrix Theory (RMT, see e.g. Onatski, 2009, 2010) that allows us to select the number of statistically significant factors estimated via Principal Component Analysis (PCA). Compared to traditional static factor analysis, our procedure does not require the introduction of penalty functions and/or ad-hoc truncation strategies to identify the number of significant factors (see e.g. Ludvigson and Ng, 2007; Guo et al., 2018). It only needs a comparison between empirically estimated eigenvalues and a distribution of eigenvalues that is generated by a Gaussian random model with only spurious correlations.

By employing the above procedure, we first characterize the Eurozone government bond yields dynamics. In addition, we provide a formal interpretation of observed patterns based on portfolio theory. We find that synchronization in yields is scarce at one-year maturity, as the most relevant factor explains around $30 \%$ of the total variance, and it is not statistically significant at specific time windows. The corresponding eigenvector components are also heterogeneous over the whole 20032019 period. The situation is different for 5-years and 10-years government bond yields. One factor explains between $75 \%$ and $80 \%$ of total variance of those yields until to 2008 ; around $40 \%$ between 
2008 and 2014; and about $60 \%$ from 2015 to 2019 . The associated eigenvector components contribute in equal amount to the dynamic of the first factor until 2008, indicating a strong synchronization between 2003 and 2008. Starting from 2008, instead, the eigenvector components of some "core" countries keep contributing to the dynamic of the first factor, while the eigenvector components of a second group of countries (the "periphery") contribute only to a low extent. Moreover, a second factor becomes significant during the crisis, accounting for a share between $20 \%$ to $30 \%$ of the total variance. Its eigenvector components constantly differ in sign across core- and peripheral countries.

Recent results in portfolio theory (see Avellaneda and Lee, 2010; Bouchaud and Potters, 2015) show the equivalence between PCA-estimated factors and the relative variance of mutually independent portfolios. Moreover, the components of eigenvectors associated to each factor correspond to the weights of assets within one portfolio. On these grounds, finding that eigenvector components diverge in sign indicates the presence of optimal trading strategy wherein some bonds are held on a long position while some others on a short one. These "divergence trades" (Avellaneda and Lee, 2010) might cause significant asymmetries in government bonds yields within a monetary union and pose debt sustainability problems for some countries.

Our results deliver new insights for the Eurozone policy debate. It is well known that the heterogeneous response of different countries' yields to the same ECB interest rate policy is one of the main fragilities for the Euro area. Our procedure allows one to quantify asynchronicity in yields' movements and, being based on daily data, it can be useful to policy makers to quickly understand whether a conventional policy can be effective or not. Under diverging government bond yields, in fact, the conventional monetary policy results in asymmetric effects across the Eurozone and the unconventional monetary policy should be used accordingly.

The paper is organized as follows. Section 2 summarizes results from previous studies that are related to our work. The data are presented in Section 3, together with a preliminary statistical descriptive analysis. Section 4 describes the econometric procedure we use in the paper. Baseline results for one-year, five-years and ten-years yields are presented in Section 5. A series of robustness checks, controlling for sample selection, model parameters, as well as non-Gaussian null models are discussed in Section 6. Section 7 discusses the results and its policy implications. Section 8 concludes. 


\section{Some methodological roots}

Our work is related to two empirical research strands. First, we refer to the bulk of studies that have investigated macroeconomic time series by using factor models and principal component analysis (PCA). The work by Stock and Watson (2002) is among the first studies that estimates static factors by means of PCA, showing that the obtained estimators are consistent and efficient as the number of time series and their length grow to infinity. ${ }^{1}$ Several works have also proposed methods to select the number of factors. For instance Bai and $\mathrm{Ng}(2002,2008)$ introduce factor selection based on penalty functions, to consistently estimate the optimal number of factors that shall then be included in a structural vector autoregressive model. Ludvigson and $\mathrm{Ng}$ (2010), estimate eight macroeconomic factors and interpret them on the basis of the marginal R-squared obtained by regressing all the variable of their dataset of the factors taken one at a time. Onatski (2010) and Kapetanios (2010) provide an alternative selection criteria of factors, based upon applications of Random Matrix Theory (RMT). In particular, Onatski (2010) shows that selection based on RMT performs better when the variance attributed to the factors is small relatively to the variance caused by the idiosyncratic components. Kapetanios (2010) relaxes some of the RMT assumptions and finds that the method is still robust to these violations. This suggests not only that RMT performs better, but also that its assumptions are not necessarily stricter than the ones required by alternative static factors estimators.

PCA has also been extensively applied to portfolio analysis in finance. For instance, Avellaneda and Lee (2010) show the existence of a direct relation between optimal portfolio theory and principal components. They compare the Sharpe-ratio performances of exchange traded funds (ETF) and portfolios weighted by principal components - i.e. the so called eigenporfolios. ${ }^{2}$ The study is conducted on the U.S. equity market from 1997 to 2007. The authors find that the portfolios that maximize the Sharpe-ratio, are given by considering either fifteen exchange traded funds, or the first fifteen principal components, or a variable number of principal components accounting for approximately $55 \%$ of the total variance. ${ }^{3}$ Following a similar approach, Guo et al. (2018) propose a selection criteria

\footnotetext{
${ }^{1}$ A notable application of static factors to the analysis of bond risk premia is the one by Cochrane and Piazzesi (2005). They regress the excess one-year bond return on a factor constructed from a linear combination of five forward spreads and find that a single factor predicts 1-year excess returns on 1-5 year maturity bonds with up to $43 \%$.

2"An ETF is an investment vehicle, with a specific architecture that typically seeks to track the performance of a specific index” (see Lettau and Madhavan, 2018).

${ }^{3}$ The article shows that a portfolio weighted by the exchange traded funds dominates the portfolio weighted by the
} 
to maximize Sharpe-ratio eigenportfolios. They use a LASSO-based factor selection, in which the threshold for the truncation is computed by introducing a function for the maximum tolerance for the relative approximation error in the estimation of the empirical correlation matrix. ${ }^{4}$

Second, our work is related to the large literature that has investigated interest rates convergence by using various econometric procedures. ${ }^{5}$ Vajanne (2007) studies the integration of retail banking rates in the Eurozone between 2003 and 2006 using a monthly data panel and adopts beta- and gamma-convergence as measures of convergence. Its findings are in favor of some convergence, notwithstanding important substantial cross-country differences. Furthermore, Arouri et al. (2013) consider interbank interest rates for France, the UK, and the US between 2004 to 2010 and use the Geweke Contemporaneous Feedback Measure (see Geweke, 1982) as a measure of convergence. ${ }^{6}$ Such a measure consists in a log-likelihood ratio test statistic, which tests whether a model with interdependent interest rates is significantly different from a model wherein interest rates depends only on their past values. The study finds evidence of convergence among the countries examined, with changes in US interest rates being slightly leading with respect to those in France and in the UK. In addition, the work by Wälti (2011) estimates the effect of a common factor (capturing trade and financial integration) on stock market returns cross-correlation. ${ }^{7}$ The work considers yearly data for 15 developed countries from 1975 to 2006 and it shows that trade and financial integration have a positive effect on stock market correlation. Nevertheless, for Eurozone members the above effect is significant only for countries that were already tightly connected before the introduction

principal components in some periods, while the opposite holds for some other periods. The performance of the two types of portfolios is nonetheless comparable and the alternating dominance of one of the two is never striking. It can be observed, accordingly, that portfolios weighted by principal components do not outperform alternative portfolios strategies, but that principal components strategies are comparable and sometimes preferable in terms of Sharpe-ratio maximization to alternative portfolio strategies.

${ }^{4} \mathrm{PCA}$ has also been applied to the analysis of systemic risk in financial markets and to business cycle synchronization (see Guerini et al., 2019). In particular, Billio et al. (2012) include principal components extracted from financial series as measures of systemic risk and early warnings indicators. Kritzman et al. (2011) focus on the fraction of total variance explained by the main factors, extracted from PCA, as an index of systemic risk.

${ }^{5}$ In this paper we focus on synchronization. While convergence is defined the tendency of different series to reach a unique value, synchronization only implies that the different series are moving together, for instance increasing or decreasing together, notwithstanding possible differences in absolute values. Synchronization might be seen in this sense as a milder form of convergence.

${ }^{6}$ Data for France are European data from the ECB. In the study, therefore, France stands as a representative country for the Eurozone in general.

${ }^{7}$ In his analysis, the common international factor consists of weekly averages of stock market indexes, the intensity of trade is estimated using a gravity model, the financial integration is represented by a measure of foreign assets that are domestically owned, and the exchange rate volatility, a dummy for being a member of the European Stability Mechanism, and a dummy for being a member of the European Monetary Union account for the monetary integration. 
of the common currency. Finally, Dow et al. (2012) analyze convergence in interest rates across Italian regions. The authors employ quarterly data on short- and long-term interest rates on loans and deposit from 1998 to 2008. Methodologically, convergence is examined through different unit root tests, where some evidence of convergence is considered if the cointegration hypothesis is not rejected.

We contribute to the above two streams of literature by performing an analysis of synchronization in Eurozone government bond yields that combines PCA with a factors selection method based on RMT. This combination allows one to precisely define and to measure the degree of synchronization in bond yields. Our procedure selects factors ex-ante on the basis of a comparison with alternative null models rather than ex-post on the basis of ad-hoc selection criteria, and it quantifies co-movements among variables of interest by studying in detail the eigenvalues and eigenvectors of correlation matrices. This is a significant difference with respect to previous works (e.g. Bai and $\mathrm{Ng}, 2002$, 2008; Ludvigson and Ng, 2010) that have employed ex-post selection methods based on factors' forecasting performance. Our approach to factor selection is guided by the consideration that a factor, when used as regressor in a forecasting model, might hypothetically turn out to be significant even if the information it contains is indistinguishable from that obtained from a spurious relationship obtainable with a finite number of observations. In addition, the eigenvalues and the eigenvectors selected through RMT provide a more precise and accurate information about synchronization of bond yields compared to the information obtained from a mere comparison of correlation coefficients. This is because it is possible to theoretically interpret significant eigenvalues as optimal portfolios and the associated eigenvectors as the weights of the assets therein (see Avellaneda and Lee, 2010).

\section{Data description and preliminary analysis}

We retrieve from the Bloomberg platform the time series of government bonds yields at daily frequency for 11 Eurozone countries and at 11 maturities. Our data include time series of bonds with maturity at 3 and 6 months as well as bonds at 1,2,3,5,7,10,15,20, and 30 years maturity. The selected Eurozone countries are Austria, Belgium, Finland, France, Greece, Germany, Ireland, Italy, Netherlands, Portugal, and Spain. Luxembourg is missing because of data unavailability problems 
in the Bloomberg platform. All countries are member states of the Eurozone since its creation with the exception of Greece, which joined the Euro area in $2001 .^{8}$ The dataset covers the period from 2003-01-01 to 2019-02-21. We focus our analysis on government bond yields with 1-year, 5-years, and 10-years maturities. These are available for all countries (with the exception of the Netherlands for 1 year bonds) over the whole sample period. The other maturities are used as robustness check, to complement our understanding of the problem (see Section 6.1).

We focus on nominal rather than real interest rates. There are several reasons underlying such a choice. First, there is a time mismatch between the reactions of nominal interest rate and inflation rate to monetary policy shocks, with inflation adjusting at a slower pace (Christiano et al., 1999). Second, nominal interest rates are directly affected by monetary policy shocks, while inflation depends on a wider range of factors, also beyond monetary policy (see e.g. Reis and Watson, 2010; Coibion and Gorodnichenko, 2015; Blanchard, 2016). Our core dataset comprises the time series for three maturities of government bond yields for $N=11$ countries and for $T>4000$ periods (see Appendix A.1 for further details).

Augmented Dickey-Fuller (ADF) tests for presence of unit roots suggest that all the time series in our sample are at least $I(1)$. Thus, before applying the RMT procedure, we perform a pre-whitening of the data by taking a first difference transformation. The series resulting from each transformation are all stationary and no structural break can be identified. ${ }^{9}$

\section{Methodology}

Our econometric strategy to investigate synchronization in bond yields exploits Random matrix theory (RMT) to select the number of statistically significant principal components based on the

\footnotetext{
${ }^{8}$ For some maturities, we postpone the beginning of the series due to a lack of observations before specific dates. In addition, not all bond maturities are available for all countries. See Appendix A.1 for a detailed description of these two issues.

${ }^{9}$ We also performed a cointegration analysis (by employing Johansen's procedure) to detect cointegration relationships among government bond yields of different countries at the same maturity. We also checked whether these relationships changed over time, by previously identifying relevant dates over which divide our time series on the basis of clustered structural breaks analysis (K-Means Cluster Analysis and Ward Hierarchical Clustering). The results indicate that the order of cointegration increased after 2008 for all the three yield maturities considered in our sample. We conclude that the number of common trends has increased after the strains of Great Recession and of the European Debt crisis. However, this analysis does not allow us to interpret the cointegration relations as co-movements among time series. Indeed, common trends are mere linear combinations of our non-stationary time series. They do not provide information about whether the time series belonging to the same linear combination also evolve together. Nonetheless, the increasing number of common trends suggests already a possible diverging dynamic in bond yields after 2008.
} 
comparison between the empirically estimated correlations of bond yields and the theoretical correlations generated by a random normal model (Laloux et al., 1999, 2000).

\subsection{Principal Component Analysis and Eigenportfolios}

We perform a Principal Component Analysis (PCA) on overlapping rolling windows of our stationary time series. A rolling window $\underset{N \times K}{X}$ is a section of length $K<T$ of our dataset $\underset{N \times T}{X}$ of stationary time series. When moving from a rolling window to the next one observations are updated by a step $S$, i.e. $S$ observations are discarded at the beginning of the window and $S$ observations are added at the end.

\begin{tabular}{cc}
\hline Number of observations & Window's time span \\
\hline 22 & Month \\
65 & Quarter \\
130 & Semester \\
261 & Year \\
522 & Two years \\
783 & Three years \\
\hline \hline
\end{tabular}

Table 1: Windows dimensions for daily Bloomberg time series of government yields.

By construction, the smaller is the window the more accurate is the tracking of synchronization. At the same time, windows' length should also be sufficiently large to ensure statistical significance of the correlation coefficients to be computed and, since we employ daily data, to obtain results which are not driven by daily outliers. We fix the length of each window to one semester (i.e. 130 daily observations) and the length of each step of the windows to one month (i.e. 22 daily observations, see Table 1 for the number of observations for each window's time span). As a result, two subsequent rolling windows overlap for 5 months out of 6 . Such a choice allows us to closely track the evolution of the principal components and their dynamics over time, by replacing only few information at each step. However, in Section 6.2 we also check the robustness of our main results to several alternative values of the length and step parameters of the rolling windows.

For each window, we compute the matrix of Pearson's correlation coefficients of bond yields, $E$ as:

$$
E=\frac{1}{K} \tilde{X}^{T} \tilde{X}
$$


where $\underset{N \times K}{\tilde{X}}$ is the demeaned and standardized version of $\underset{N \times K}{X}$ and $(\cdot)^{T}$ is the transpose operator. The matrix $E$ has size $N \times N$. Its element $e_{i, j}$ is the correlation coefficient between the government bonds yields of countries $i$ and $j$ in a specific window $w \in\{1, \ldots W\}$. From this positive semidefinite matrix it is possible to compute the eigenvalues, which are all non-negative and distinct, and their associated eigenvectors. Each eigenvalue $\lambda_{i}$ corresponds to a principal component, or a factor, and it is a linear combination of the original series that explains a specific portion of the variance contained in the data.

Furthermore, each eigenvalue has a precise financial interpretation. More precisely, consider a portfolio $\Pi_{i}=\tilde{X} u_{i}$, where the normalized returns $\tilde{X}$ are weighted by a vector $u_{i}$. The variance of such a portfolio is equal to:

$$
\operatorname{Var}\left(\Pi_{i}\right)=\operatorname{Var}\left(\tilde{X} u_{i}\right)=E\left[\tilde{X} u_{i}\right]^{2}=\frac{1}{K} u_{i}^{T}\left(\tilde{X}^{T} \tilde{X}\right) u_{i}
$$

By using the spectral decomposition we can write equation 1 as:

$$
E=u_{i} \lambda_{i} u_{i}^{T}
$$

and by substituting it into equation 2 , it follows that

$$
\frac{1}{K} u_{i}^{T}\left(\tilde{X}^{T} \tilde{X}\right) u_{i}=u_{i}^{T} E u_{i}=\lambda_{i}
$$

Equation 4 thus shows that each eigenvalue $\lambda_{i}$ corresponds to the variance of returns - and thus to the risk - of the eigenportfolio $\prod_{i}$, composed by the $N$ different government bonds. The weights of each bond in the portfolio are returned by by the elements of the corresponding eigenvector (see Bouchaud and Potters, 2015). In particular, a larger eigenvalue, corresponds to a higher risk of the corresponding eigenportfolio. It follows that the eigenportolio associated with the dominant eigenvalue of the correlation matrix is also the one that maximizes risk. ${ }^{10}$

\footnotetext{
${ }^{10}$ This portfolio is also known in the literature as the "market mode" portfolio (see Bouchaud and Potters, 2015).
} 


\subsection{Random Matrix Theory and Factors Selection}

Random Matrix Theory (RMT) allows one to select only the principal components that contain information that is not entirely reducible to spurious volatility. ${ }^{11}$ For spurious volatility here, we do not refer to the correlation of two $I(1)$ variables, but to the idea that even two stationary i.i.d variables with a finite number of observations can display some degree of correlation. The important question thus becomes how to distinguish whether the distribution of the observed empirical correlations is different from the one that would have observed for finite i.i.d variables. RMT solves this problem by comparing the empirical correlation matrix with the correlation matrix of a randomly generated normal model.

Marčenko and Pastur (1967) theorem plays a central role in RMT. This theorem states that the probability density function of the eigenvalues of a random correlation matrix derived from normal independent and identically distributed series, is distributed according to the Marčenko-Pastur distribution (see Marčenko and Pastur, 1967; Laloux et al., 1999)).

Theorem - Marchenko-Pastur law. For $N, T \rightarrow \infty$ and $Q=\frac{T}{N} \rightarrow a>1$, the density function of the eigenvalues of $\hat{\Sigma}$ is given by

$$
\rho \hat{\Sigma}(\lambda)= \begin{cases}\frac{Q}{2 \pi \sigma^{2}} \frac{\sqrt{\left(\lambda_{m a x}^{r m t}-\lambda\right)\left(\lambda-\lambda_{\min }^{r m t}\right)}}{\lambda} & \text { for } \lambda \in\left(\lambda_{\min }^{r m t}, \lambda_{\max }^{r m t}\right) \\ 0 & \text { else }\end{cases}
$$

where $\lambda_{\max / \min }^{R M T}=\sigma^{2}\left(1 \pm \sqrt{\frac{1}{Q}}\right)^{2}$ are the upper/lower bounds of the eigenvalues associated with a random matrix with the same variance $\sigma^{2}$ and the same $Q$ of the empirical observations.

Thus, any empirical eigenvalue lying within the boundaries of the theoretical Marčenko-Pastur distribution explains a fraction of the variance that is comparable to the one generated by a purely random model where correlations are only spurious. In contrast, eigenvalues lying outside such boundaries contain some pure information about the underlying stochastic process governing comovements among the variables of interest. Since the Marčenko-Pastur law holds only under asymptotic assumptions, and our dataset is particularly limited in the country dimension (small N), we

\footnotetext{
${ }^{11}$ Since factor models can be estimated through principal components (Stock and Watson, 2002; Onatski, 2010), our method can also be used for factor selection. The factor analysis is discussed in Appendix B.
} 
compute the Marčenko-Pastur boundaries using Monte Carlo simulations and we compare our empirically estimated eigenvalues to the simulated ones. Furthermore, we carry out several robustness checks on the Marčenko-Pastur boundaries (i) by applying a rotational random shuffling procedure to our time series in order to clean the results from the possible residual autocorrelations present in the stationary time series (see Section 6.4); (ii) by adopting a heavy tails random i.i.d. modes which that accounts also for the possibility of extreme values in the yields series (see Section 6.5).

\section{Results}

Let us now present the main results of our empirical analysis of government bond yields in the Eurozone. We first present some statistics about the dynamics of bond yields correlation in our sample (Section 5.1). These statistics deliver first insights about the nature of yields comovements in the Euro Area. Yet, they are only an imperfect measure of yields synchronization because they do not account for the presence of spurious correlation. In Section 5.2, we then present the results obtained using our preferred measure of synchronization that combines PCA and RMT, and which allows us to filter out the spurious (noisy) information.

\subsection{The network of government bond yields correlations}

The empirical correlation matrix among government bond yields $E$ that we analyze (See Equation (1) above) is a combination of a non-spurious correlation component and of a spurious idiosyncratic component - i.e. $e_{i, j}=c_{i, j}+\chi_{i, j}$. Following Tumminello et al. (2010) and Diebold and Yilmaz (2014), this matrix can be interpreted as a weighted undirected graph where the vertices represent the stationary normalized yields and the link weights measure their correlations (see also Diebold and Yilmaz, 2014).

Figure 1 visualizes the structure of some of the above-defined correlation networks (from 2008 to 2011) for bonds with 10 years maturity. The thickness of the links in the networks is proportional to the magnitude of correlations. Negative correlations are depicted in red.

Overall, strong and positive correlations among all yields were present up until 2008. From 2009 onward, instead, average correlation in yields decreases and it becomes even negative in some 


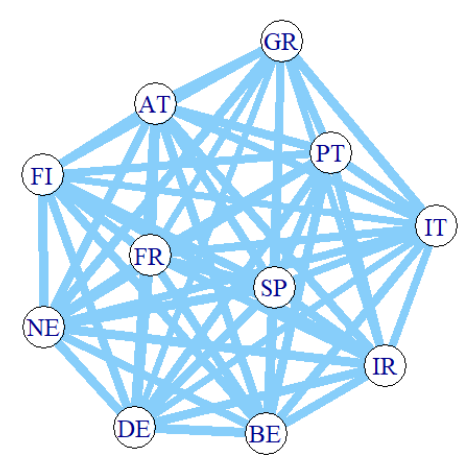

(a) 2008

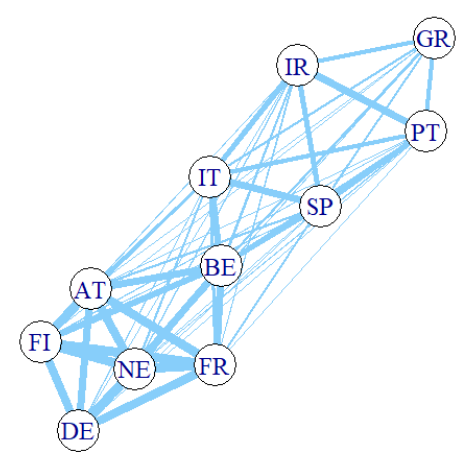

(c) 2010

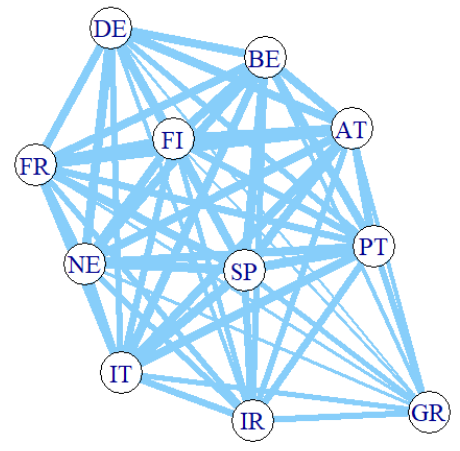

(b) 2009

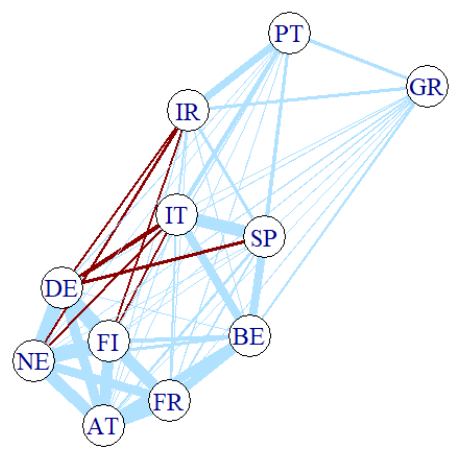

(d) 2011

Figure 1: The structure of the bond yields correlation networks from 2008 to 2011. Government bonds with 10-years maturity. The thickness of the links is proportional to the magnitude of the correlations. Positive correlations are lightcoloured (sky blue), negative correlation are dark-coloured (red). Countries: AT = Austria, BE = Belgium, FI = Finland, $\mathrm{FR}=$ France, $\mathrm{DE}=$ Germany, GR $=$ Greece, $\mathrm{IR}=$ Ireland, $\mathrm{IT}=$ Italy, NE $=$ Netherlands, $\mathrm{Pt}=$ Portugal, $\mathrm{SP}=\mathrm{Spain}$.

cases. Two clusters of countries can be identified in this period. A first group is composed by "core" countries, and it mainly includes Northern countries like Germany, the Netherlands, Austria and Finland. These countries have strong positive correlations among their yields. In contrast, the bond yields of a second group countries (the "periphery"), including Greece, Ireland, Portugal, Spain and Italy, displayed weak of even negative correlations with all the other yields. It must be stressed that negative yields correlations disappeared after 2015 (not shown in figure) and that the magnitude of yields correlation generally increased, though not recovering the high levels displayed before $2008 .{ }^{12}$

We also quantify how much the structure of correlation networks has changed over time. For this purpose, and following Münnix et al. (2012), we build a similarity index that measures how much the correlation adjacency matrix $E$ in a specific time window $w_{1}$ is similar to the correlation

\footnotetext{
${ }^{12}$ This is especially the case for Greece, whose yields still display a weak correlation with yields of other countries in the Eurozone.
} 
matrix of another window $w_{2}$. This similarity index is defined as:

$$
\mathcal{S}\left(w_{1}, w_{2}\right)=1-\mathbb{E}\left|E\left(w_{1}\right)-E\left(w_{2}\right)\right|
$$

it represents the average Euclidean distance between two correlation matrices. The closer the index is to one, the higher is the similarity between the network structures of two time windows. Figure 2 plots the evolution of the similarity index, normalized with respect to the first window, i.e. the one of 2003 , which we select as the reference window. ${ }^{13}$

The network of 1-year yields (Panel a Fig. 2) does not display much variation over time. The similarity index is always very high, between 0.75 to 0.9 . In contrast, the network similarity of 5years and 10-years bond yields (Panel b and c Fig. 2) decreased steadily since 2003 hitting the lowest value of about 0.5 between 2011 and 2013. This is in accordance with the previous results about the emergence of a core-periphery structure during the Great Recession and the European Debt Crisis. ${ }^{14}$

The above network representation could be affected by endogeneity problems (see e.g. Manski, 1993). For instance, the link between the yields of countries $i$ and $j$ might be affected by the fact that they are in reality correlated with the yield of a third country $m$. To control for this problem, we employ the club convergence procedure introduced by Phillips and Sul (2007). ${ }^{15}$ The procedure was originally developed for low frequency data and the main technical issue concerns the selection of the baseline period for the ordering of the time series. Our time series have more than 4000 daily observations. Accordingly, the selection of clubs might be sensible to the selection of the reference period. We tested the algorithm over different daily selections. We find that two clubs of countries are robustly present over the 2008-2012 period in line with the results discussed above. Nevertheless, the composition of the two clubs is not constant across time.

\footnotetext{
${ }^{13}$ It shall be noticed that, even if two windows are similar to the first one, they might be very different from one another. What one can say is only that they have a similar number of weights with positive and negative links, not whether the vertices of the networks of the two windows are correlated in a similar way in the two periods.

${ }^{14}$ The similarity index can additionally be used to measure the network turbulence - i.e. how fast changes in the network structure occur. Thus, we also calculated the similarity index between each window and the windows of three, six months and one year before. We find that the speed of the changes in the network structure increases before the periods of crisis, i.e. around 2007, 2009, and 2017.

${ }^{15}$ For this purpose, we use the R package Convergenceclubs developed by Sichera and Pizzuto (2019).
} 


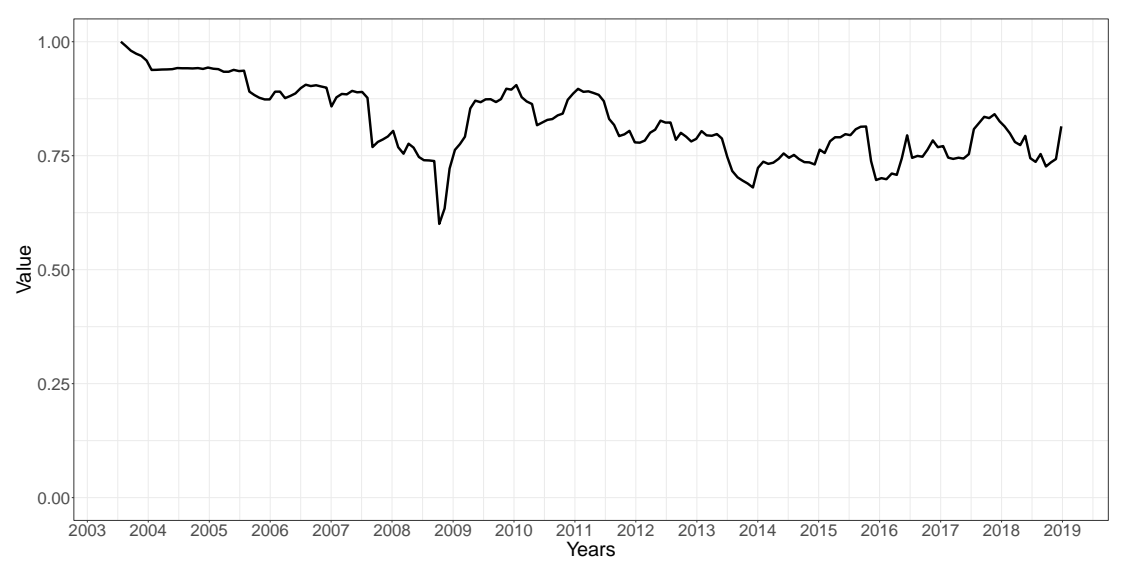

(a) One year yields

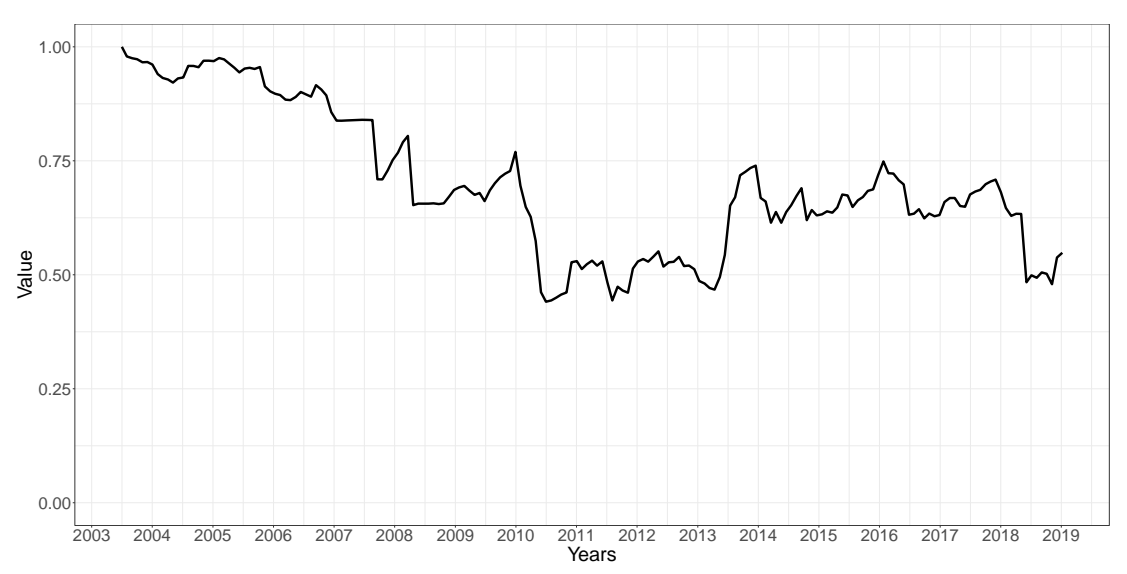

(b) Five year yields

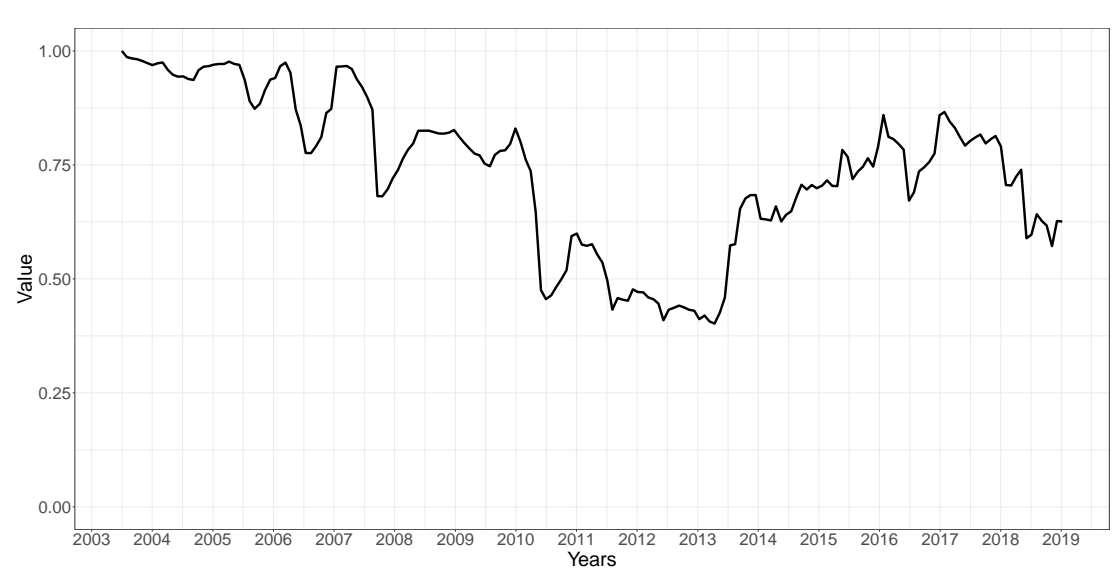

(c) Ten year yields

Figure 2: The evolution of the similarity index for bond yields correlation networks at different maturities.

\subsection{Detecting significant synchronization in government bond yields}

In the previous section, we highlighted that the network of Eurozone government bond yields correlations changed its structure during the 2003-2019 period, especially during the Great Recession and the European Debt Crisis, which resulted into divergent bond yields dynamics and in the ap- 
pearance of a core-periphery structure in the network. We now move to a more systematic analysis of bond yields synchronization by employing the methodology presented in Section 4 .

Figure 3 shows the evolution of the eigenvalues $\lambda(w)$ obtained from the spectral decomposition of each window-specific correlation matrix $E(w)$, where $w \in\{1, \ldots, W\}$ represents a specific time window. The three plots in the figure refer to the different maturities we consider ( 1 year and 5 and 10 years). Each plot also shows the different boundaries resulting from our selection procedures based on RMT, which allow one to detect significant component. Finally, each plot also allows one to compare the dynamics of eigenvalues with the timing of important events related to either shocks (e.g. Lehman Brothers' bankruptcy, which represents the start of Great Recession) or policy announcements (e.g. the famous "Whatever it Takes" speech by the ECB president Mario Draghi in July 2012 during the European Debt crisis) that have likely had an impact on bond yields (see also Section 7 for a discussion of the policy implications of our results).

As explained in Section 4.1, the higher is the component, the higher is the fraction of the timeseries bond yields variance that it can explain. The fraction of explained variance by each principal component is also called the absorption rate. These absorption rates are shown in Figure $4 .{ }^{16}$

The analysis of the plot in the top panel of Figure 3 indicates that only one non-spurious principal component explains the dynamic of 1-year bond yields. Indeed, only the largest eigenvalue is statistically significant according to RMT bounds, and only at specific moments like the Great Recession of 2008-2010, and the Eurozone debt crisis (that started with the Greek government misreport in 2009 and lasted at least until 2015). ${ }^{17}$ In addition, the dominant eigenvalue explains about $30 \%$ of the total variance except during the Great Recession period, where the share of explained variance is about $50 \%$.

In contrast to 1-year bonds, the largest eigenvalue of 5-years and 10-years government bond yields is always significant (cf. Panels b and c of Figure 3). This first principal component accounts, on average, for about $75 \%$ and $80 \%$ of the total variance before 2008 , reaching a peak of more than $90 \%$ in 2008. Nevertheless, the portion of variance explained by the dominant eigenvalue falls abruptly after 2008 to recover only in 2014. A second principal component becomes statistically significant

\footnotetext{
${ }^{16}$ The absorption rate of the $i^{t h}$ component is formally defined as $A R_{i}=\frac{\lambda_{i}}{\sum_{j}=1^{J} \lambda_{j}}$, with $i \in\{1, \ldots, J\}$. The absorption rates of statistically significant components according to RMT are shown in Figure 4.

${ }^{17}$ The misreport of public finances data by the Greek government occurred in late 2009. However, it was officially announced by the European Commission in January 2010. The plots in Figure 3 use the latter date.
} 


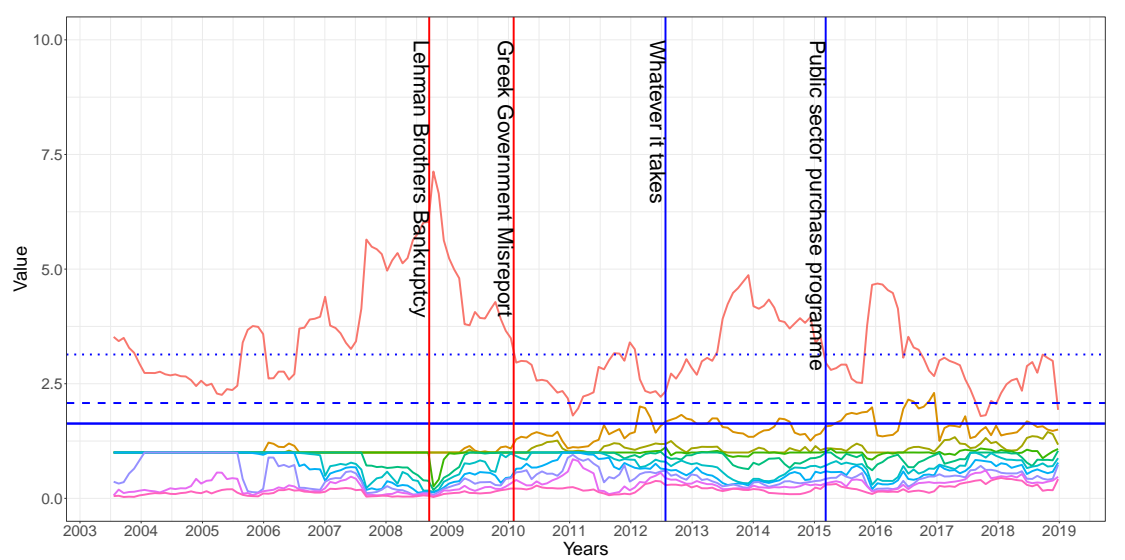

(a) One year yields

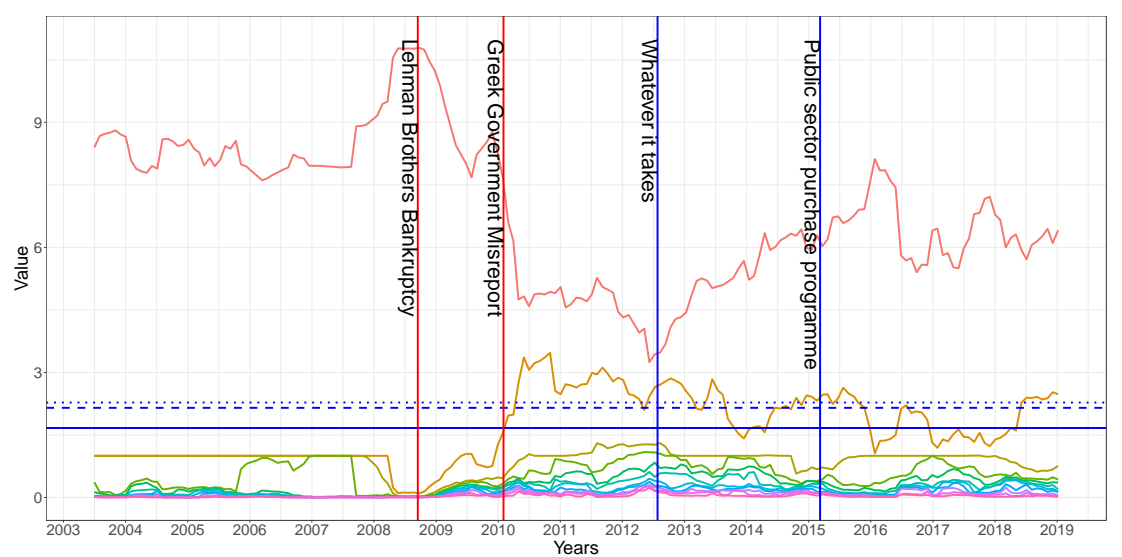

(b) Five year yields

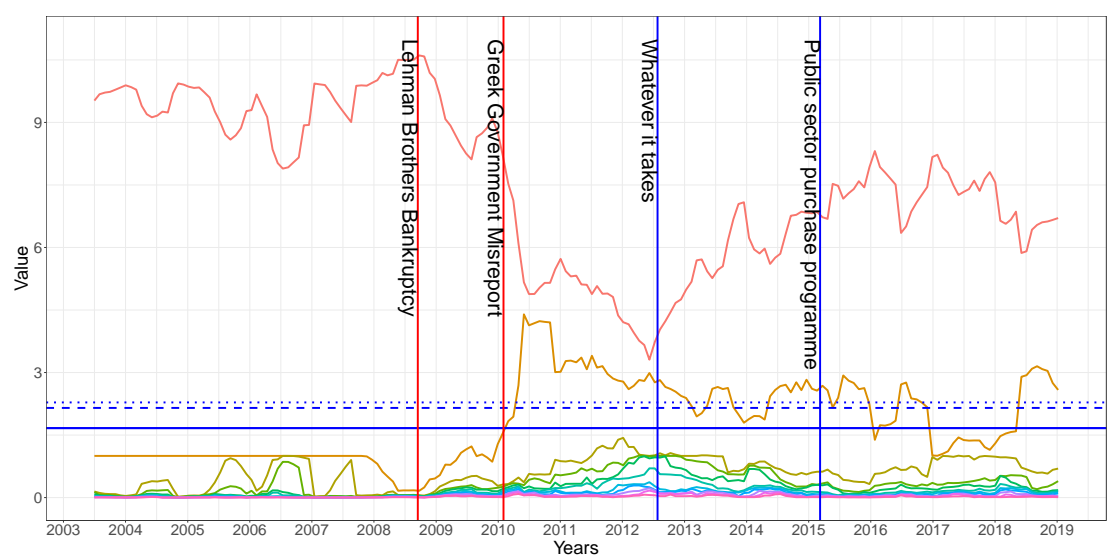

(c) Ten year yields

Figure 3: Eigenvalues evolution and multiple theoretical bounds.

Notes: the horizontal solid line indicates the Marčenko-Pastur theoretical upper bound; the horizontal dotted line indicates the Monte Carlo simulated upper bound; the horizontal thinner dotted line indicates the rotational random shuffling upper bound. For both the Monte Carlo simulated model and the rotational random shuffling, 300 Monte Carlo simulations were run. Each window of the RMT exercise contains 130 time-observations with steps of 22-observations each. 


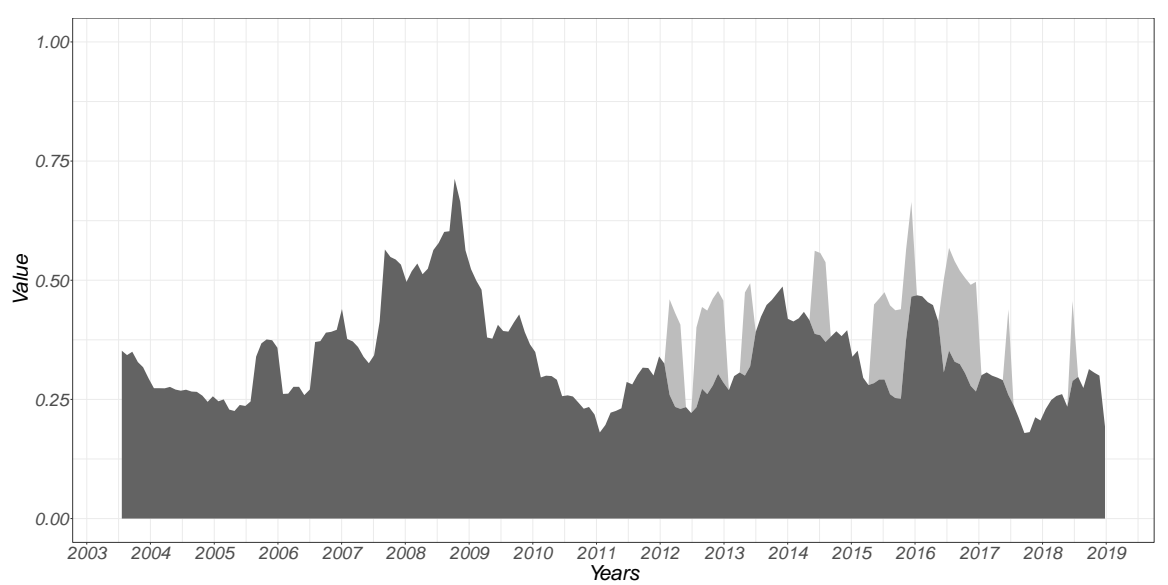

(a) One year yields

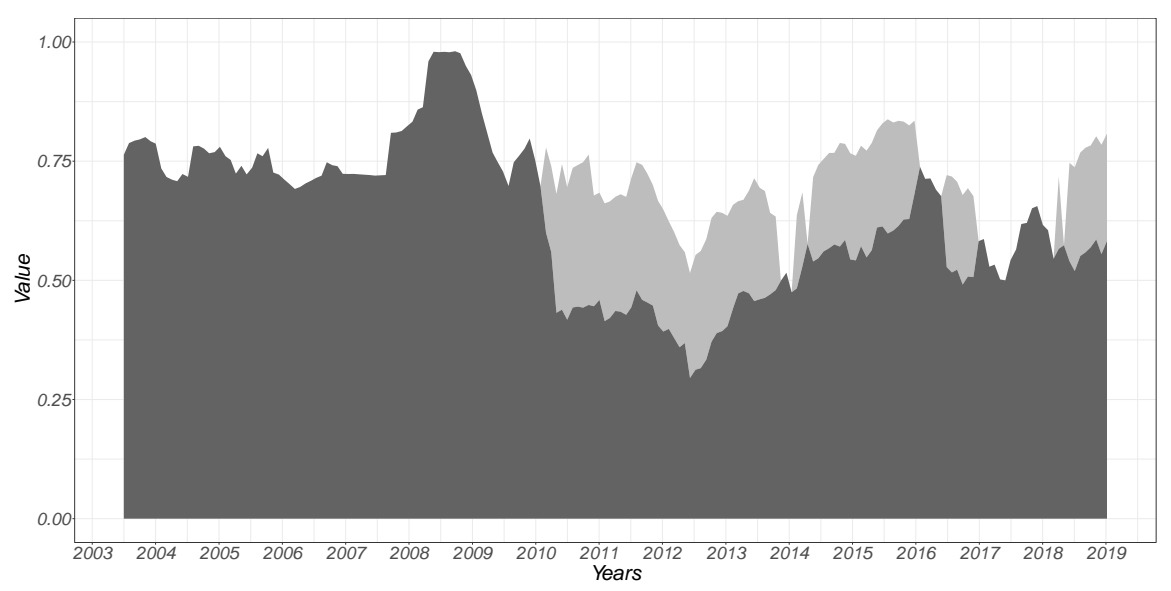

(b) Five year yields

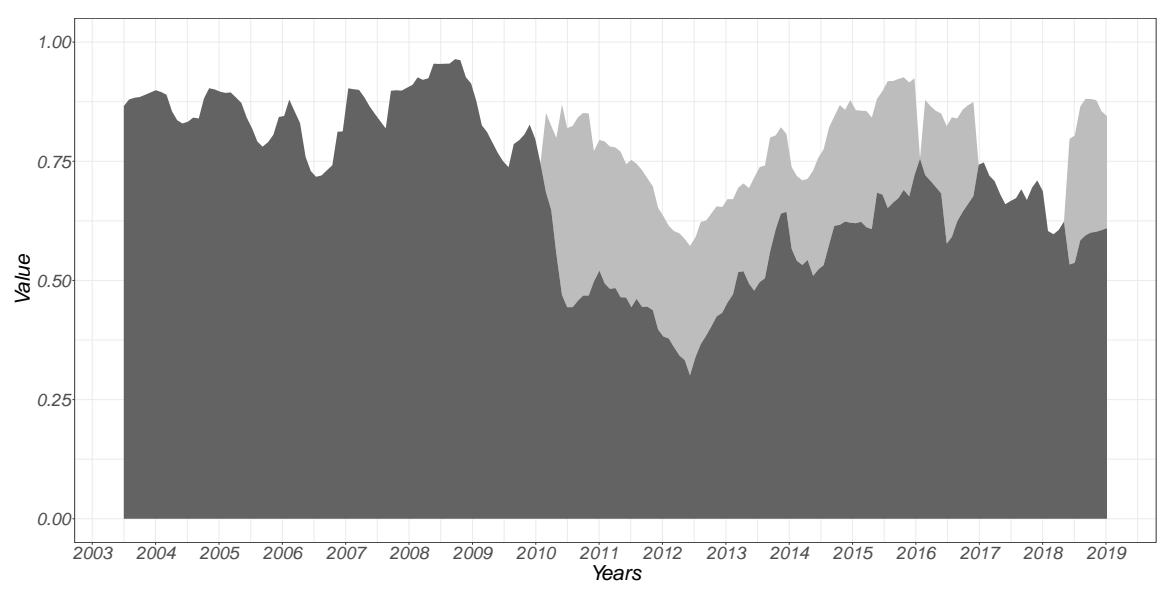

(c) Ten year yields

Figure 4: Absorption ratios for significant eigenvalues according to the RMT Marčenko-Pastur theoretical upper bound. First largest eigenvalue in dark grey, second largest eigenvalue in grey (eigenvalues normalized between 0 and 1).

in that period, and it accounts for a share between $20 \%$ and $30 \%$ of the total variance.

Finding that one principal component explains a large fraction of the total variance of bond yields is not enough to conclude that movements in those yields were synchronous. This is because 
yields can contribute in different - and possibly opposite - directions to a component dynamic. For this reasons, we also investigate in detail the components of the eigenvectors associated to each significant eigenvalue.

As we mentioned in Section 4 our approach to bond yields synchronization also involves the analysis of eigenvector components associated to each significant eigenvalue. To this end, Figure 5 shows the evolution of the elements of the eigenvector associated to the largest eigenvalue of 1-year bond yields. The dynamics of these eigenvector components is very fragmented. No group dynamics can be detected and eigenvector elements evolve heterogeneously throughout the whole sample period. We conclude that synchronization in short-term yields is much weaker than synchronization in medium- and long-term yields.

Let us now consider the eigenvectors of bond yields at 5 and 10 years, which had at least one component being significant over the whole sample period. The bar plots in Figure 6 and Figure 7 show the evolution of the elements of the eigenvector associated to the largest eigenvalue of 5-year and 10-year yields respectively between 2008 and 2014. Eigenvector elements are all very similar until 2008, documenting a high level of synchronization in bond yields. The pattern changes dramatically afterwards, and eigenvector elements start to diverge since 2009. In this respect (and like in 5.1) we can identify two groups of countries. The eigenvector components of a first group of core countries (mainly corresponding to Northern economies in the Eurozone) all display high and positive values until 2014. In contrast, the values of the elements of the other group, the periphery (Italy, Ireland, Spain Portugal, Greece), steadily decrease after 2009. Eigenvector elements even become negative for some countries like Portugal at some specific windows. The above group dynamics in bond yields is even more evident when we analyse the elements of the eigenvector associated to the second principal component, which is significant between 2008 and 2014 (see Figure 8 and Figure 9). In particular, the eigenvector elements corresponding to yields of Southern economies like Greece, Portugal, Spain and Italy are all negative between 2011 and 2015. Interestingly, between 2011 and 2013 - i.e. in the midst of the European Debt Crisis - also eigenvector elements of French and Austrian bond yields turn out the be negative. 


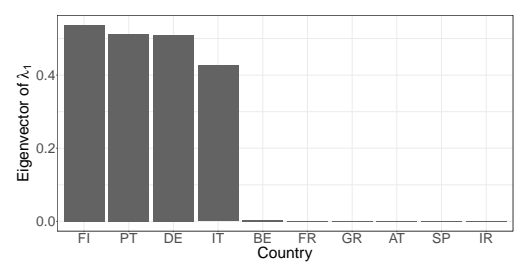

(a) 2003

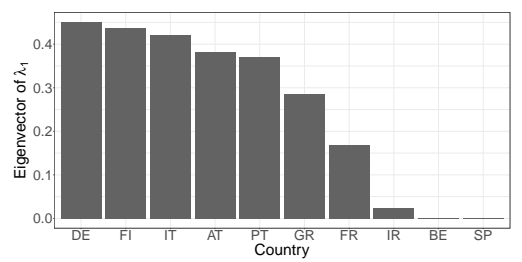

(d) 2009

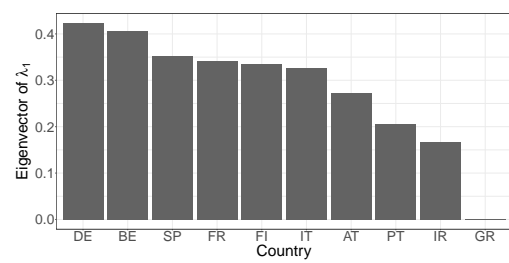

(g) 2015

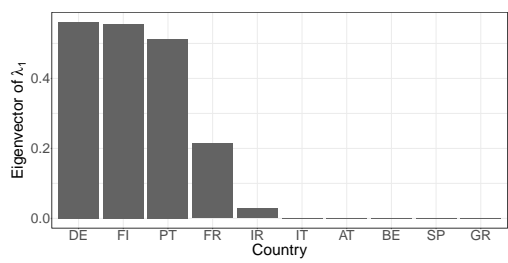

(b) 2005

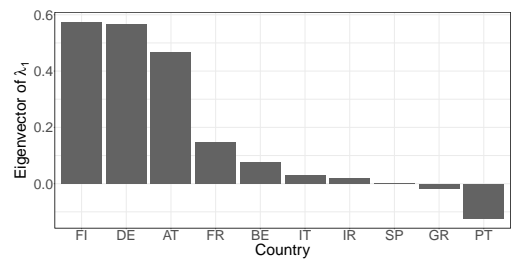

(e) 2011

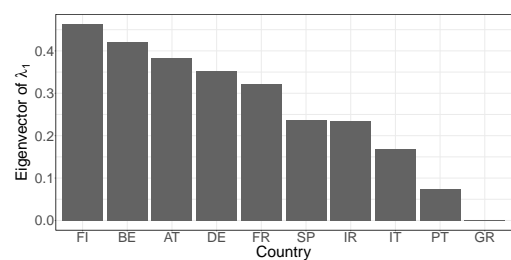

(h) 2017

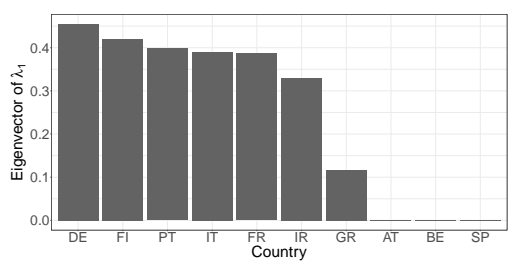

(c) 2007

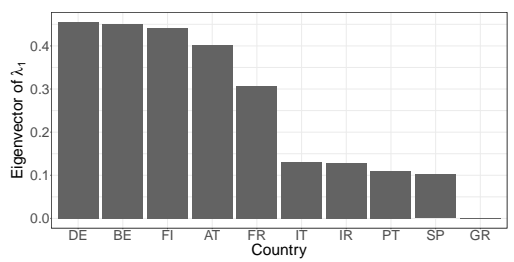

(f) 2013

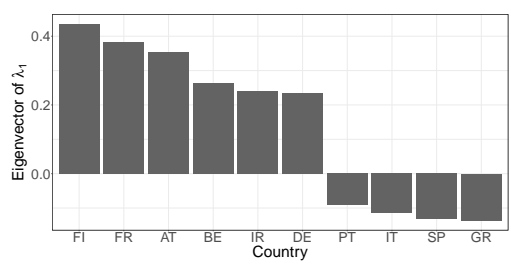

(i) 2018

Figure 5: 1-year bonds. Yearly average of eigenvector elements associated to the first principal component. Countries: $\mathrm{AT}=$ Austria, $\mathrm{BE}=$ Belgium, FI = Finland, FR $=$ France, $\mathrm{DE}=$ Germany, GR $=$ Greece, $\mathrm{IR}=$ Ireland, $\mathrm{IT}=\mathrm{Italy}, \mathrm{NE}=$ Netherlands, $\mathrm{Pt}=$ Portugal, $\mathrm{SP}=$ Spain.

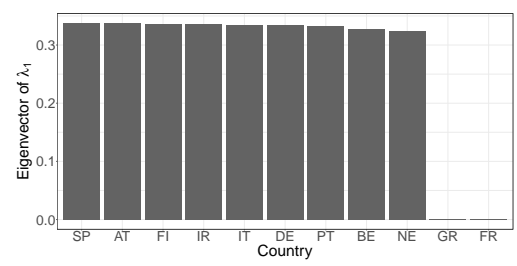

(a) 2003

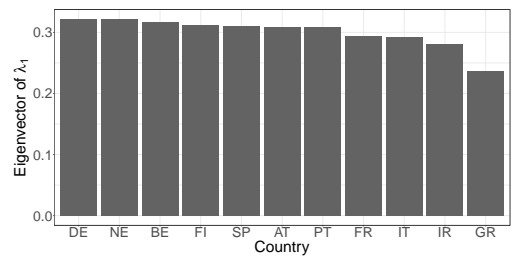

(d) 2009

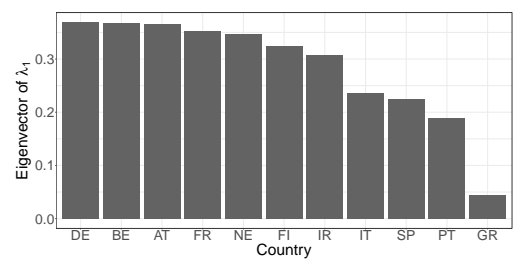

(g) 2015

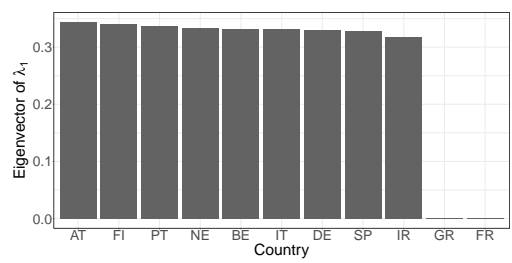

(b) 2005

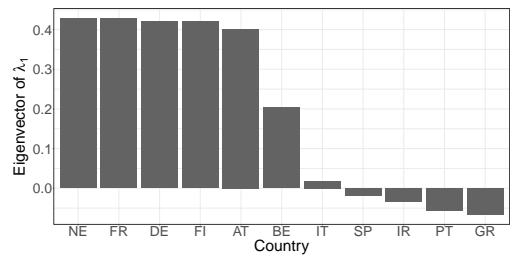

(e) 2011

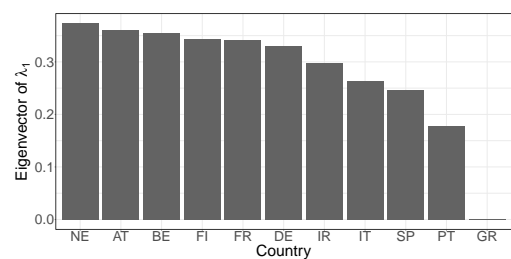

(h) 2017

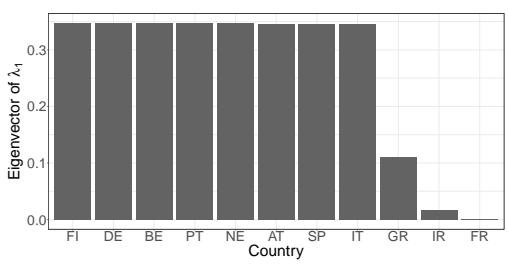

(c) 2007

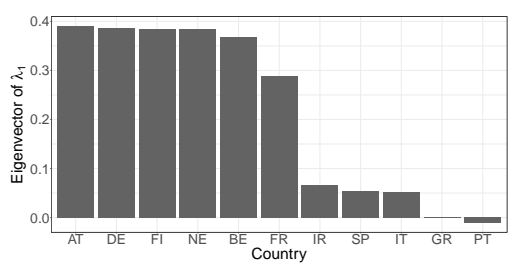

(f) 2013

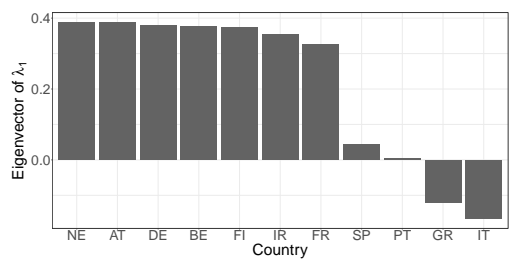

(i) 2019

Figure 6: 5-year bonds. Yearly average of of eigenvector elements associated to the first principal component. Countries: $\mathrm{AT}=$ Austria, $\mathrm{BE}=$ Belgium, FI = Finland, FR = France, $\mathrm{DE}=$ Germany, GR $=$ Greece, $\mathrm{IR}=$ Ireland, IT $=$ Italy, NE = Netherlands, $\mathrm{Pt}=$ Portugal, $\mathrm{SP}=$ Spain. 


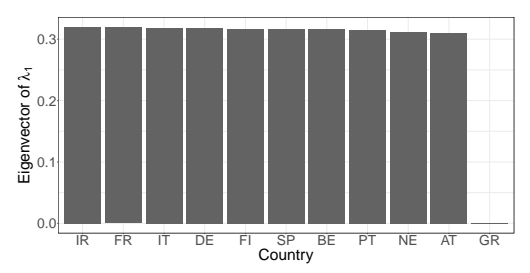

(a) 2003

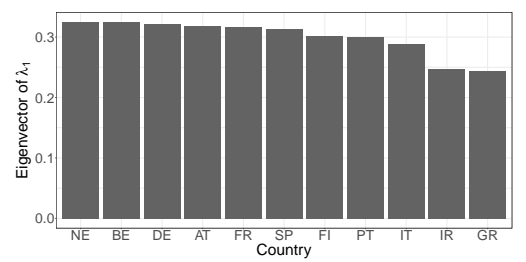

(d) 2009

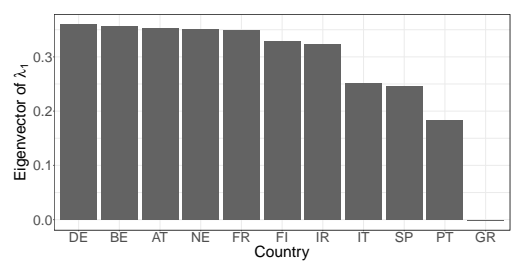

(g) 2015

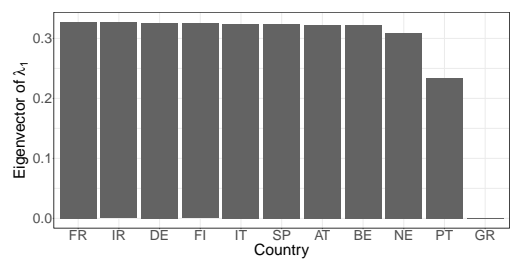

(b) 2005

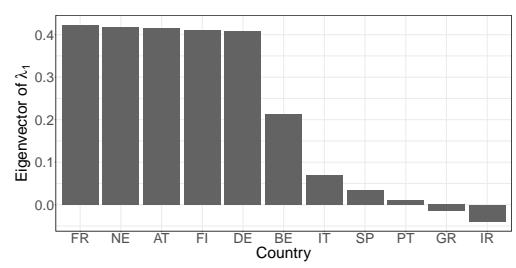

(e) 2011

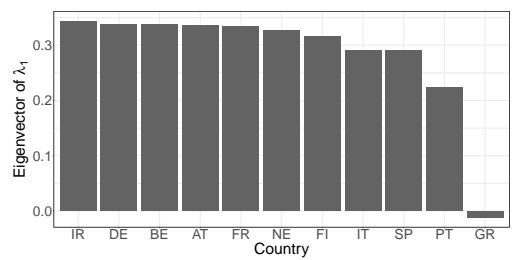

(h) 2017

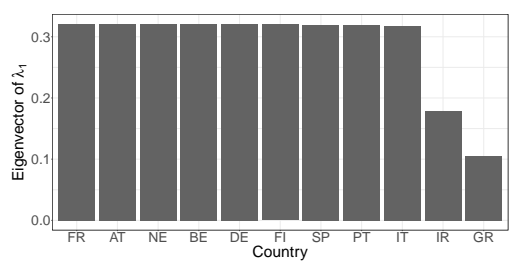

(c) 2007

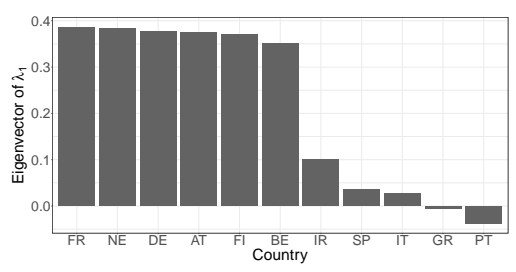

(f) 2013

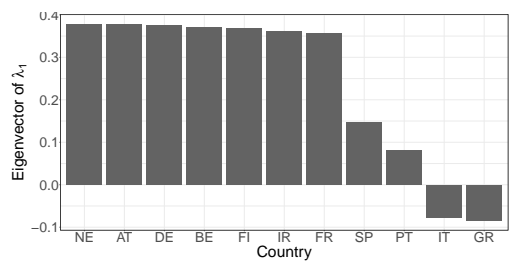

(i) 2019

Figure 7: 10-year bonds. Yearly average of eigenvector elements associated to the first principal component. Countries: $\mathrm{AT}=$ Austria, $\mathrm{BE}=$ Belgium, $\mathrm{FI}=$ Finland, FR $=$ France, $\mathrm{DE}=$ Germany, GR $=$ Greece, $\mathrm{IR}=$ Ireland, IT $=\mathrm{Italy}, \mathrm{NE}=$ Netherlands, $\mathrm{Pt}=$ Portugal, $\mathrm{SP}=$ Spain.

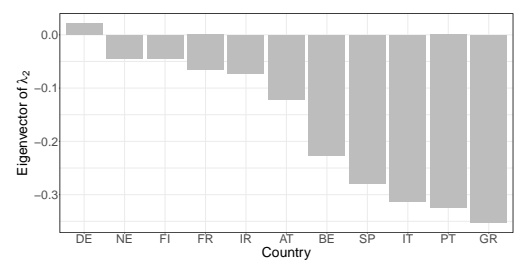

(a) 2010

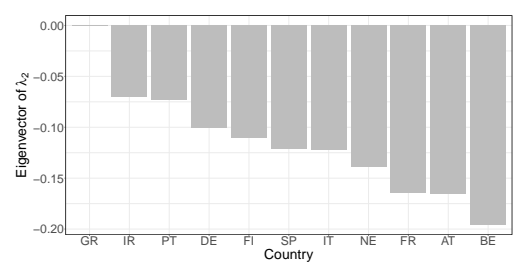

(d) 2013

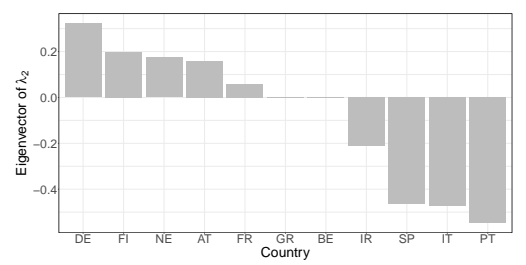

(g) 2016

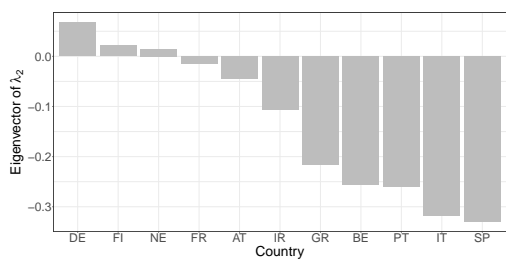

(b) 2011

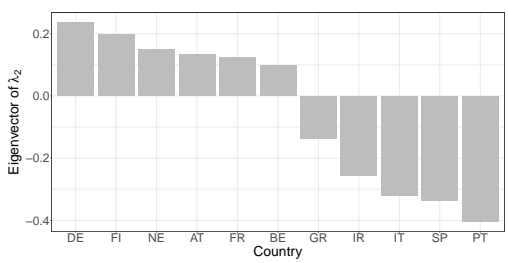

(e) 2014

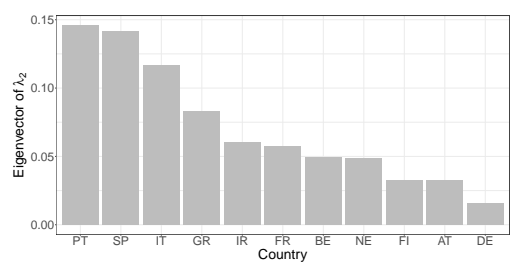

(h) 2018

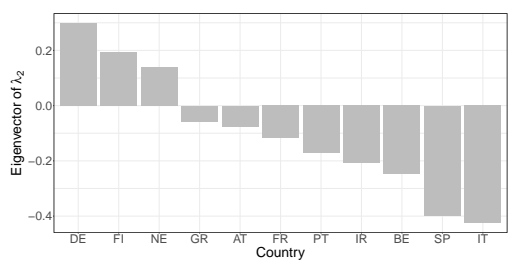

(c) 2012

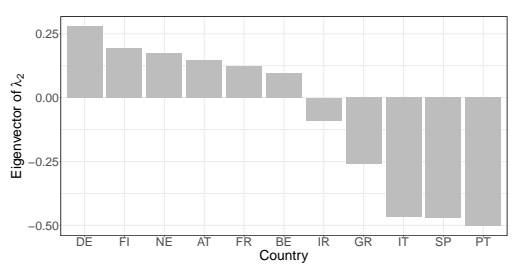

(f) 2015

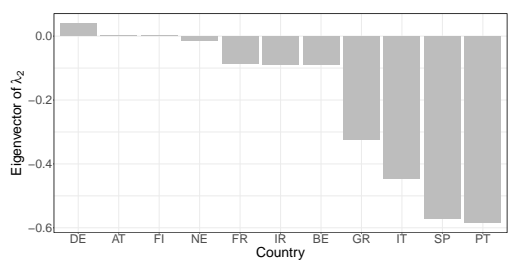

(i) 2019

Figure 8: 5-year bonds. Yearly average of eigenvector elements associated to the second principal component. Only years wherein the second principal component is significant are displayed. Countries: $\mathrm{AT}=\mathrm{Austria}, \mathrm{BE}=\mathrm{Belgium}, \mathrm{FI}=$ Finland, FR = France, DE = Germany, GR = Greece, IR = Ireland, IT = Italy, NE = Netherlands, Pt $=$ Portugal, $\mathrm{SP}=\mathrm{Spain}$. 


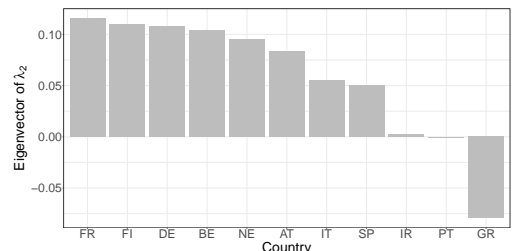

(a) 2010

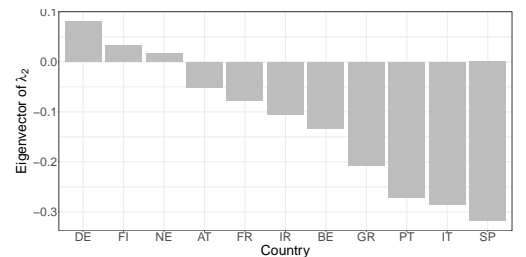

(d) 2013

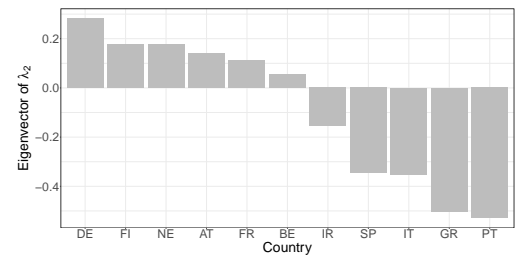

(g) 2016

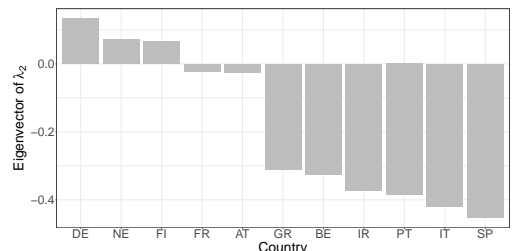

(b) 2011

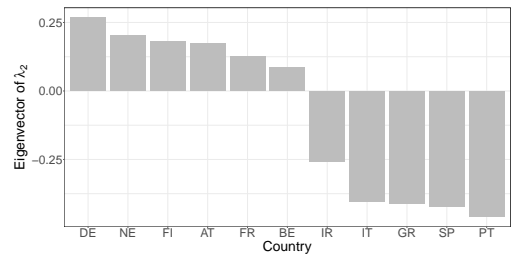

(e) 2014

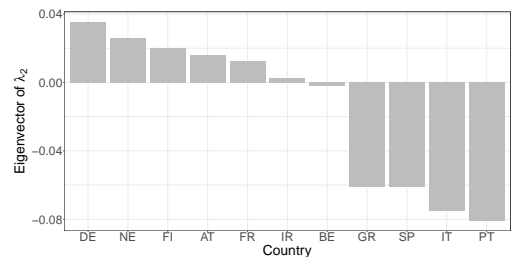

(h) 2018

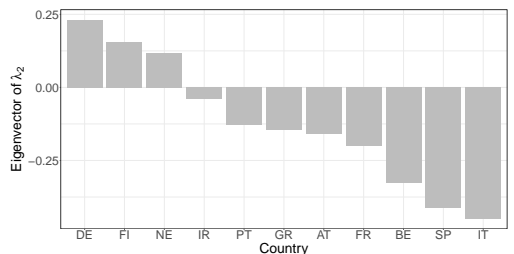

(c) 2012

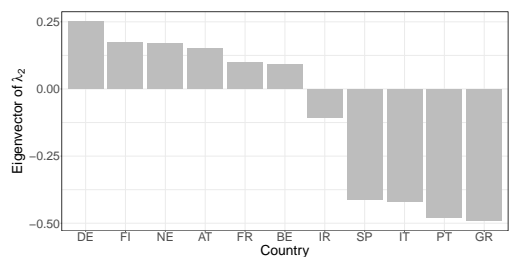

(f) 2015

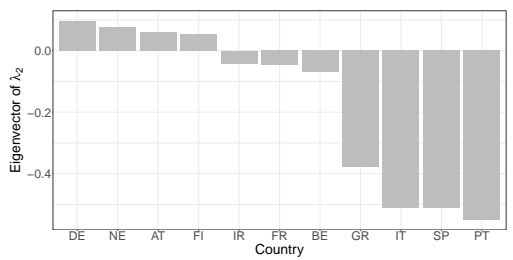

(i) 2019

Figure 9: 10-year bonds. Yearly average of of eigenvector elements associated to the second principal component. Only years wherein the second principal component is significant are displayed. Countries: $\mathrm{AT}=$ Austria, BE $=\mathrm{Belgium}, \mathrm{FI}=$ Finland, FR = France, DE = Germany, GR = Greece, IR = Ireland, IT = Italy, NE = Netherlands, Pt $=$ Portugal, $\mathrm{SP}=\mathrm{Spain}$.

\section{Robustness analyses}

The results presented in the previous section indicate that movements in short-term government bond yields are pretty much asynchronous in the Eurozone. Medium- and long-term yields were instead very synchronized until the Great Recession hit in 2008 and began to move asynchronously afterwards. This yields asynchronicity became dramatic between 2011 and 2014 with the emergence of clear divide between Northern and Southern countries in the Eurozone.

Our evidence could however be biased by at least five problems. First, the patterns we uncovered could be limited to the specific bond maturities we considered (1 year, and 5 and 10 years). Bonds at alternative maturities could exhibit very different dynamics from the one documented in the previous section. Second, our results could be affected by the length and step used to build rolling windows, as well as by the filtering technique employed (first differencing). Third, statistical significance of principal components could be biased by the presence of autocorrelation in the filtered series. Finally, statistical significance of principal components could be biased upwardly if 
the distributions of the underlying time series is heavy-tailed. In the remainder of this section we present the results of robustness checks that address all the above issues in detail. ${ }^{18}$

\subsection{Alternative yield maturities}

We perform the random matrix theory analysis on all types of bond yields available in the dataset retrieved from the Bloomberg platform. We consider yields below 1-year maturity as short-term, yields between 1-year and 10-years as medium-term; and yields above 10-years maturity as longterm (Figure 10). ${ }^{19}$

The analysis of these more comprehensive datasets confirms the main results presented in Section 5. Synchronization of short-term yields is limited and the largest component is typically nonsignificant. Furthermore, synchronization is high for medium-term maturities until 2008, it sharply falls between 2008 and 2014, to slowly recovers after 2015. However, in none of the medium-term maturities considered the degree of synchronization (See Panels b and c of Figure 3, and Panel b of Figure 10) recovered the pre-2008 levels. Finally, synchronization dynamics in longer-term yields mimics the one observable for medium-term ones. However, we find that the degree of synchronization between 2016 and 2018 of bonds with 15, 20 and 30 years of maturity is very similar to the one observed in the 2006-2009 period. At such very long time-horizons yields synchronization has fully regained the levels observed before the Great Recession.

\subsection{Alternative rolling windows}

Our econometric procedure for the analysis of synchronization involves determining rollling windows wherein correlations across bond yields are calculated. We therefore investigate the robustness of our results with respect to changes in the values of (i) the windows length $K$ and (ii) the step parameter $S$ defining such rolling windows (see Section 4 for more details). In particular, we test all the possible combinations of parameters such that $K>S$, and such that subsequent windows do overlap for at least one observation. This sensitivity analysis also encompasses combinations of pa-

\footnotetext{
${ }^{18}$ In addition to the robustness checks addressing the above issues we also repeated the analysis by computing crosscorrelations using the Spearman rank correlation coefficient rather than the Pearson coefficient. This type of control has also been used also in the literature on business cycles synchronization (see e.g. Belo, 2001). The results we obtain match (qualitatively and quantitatively) those obtained by applying the Pearson correlation coefficient.

${ }^{19}$ Data for maturities lower than 1-year and above 10-years begin only in 2008 and 2006 respectively.
} 


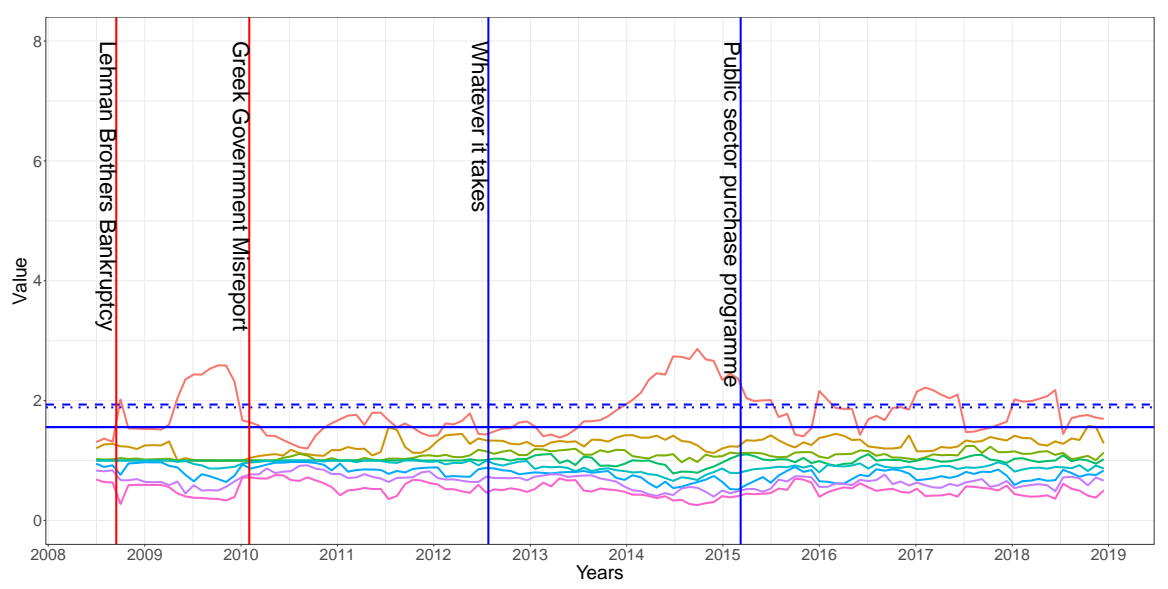

(a) Three months yields

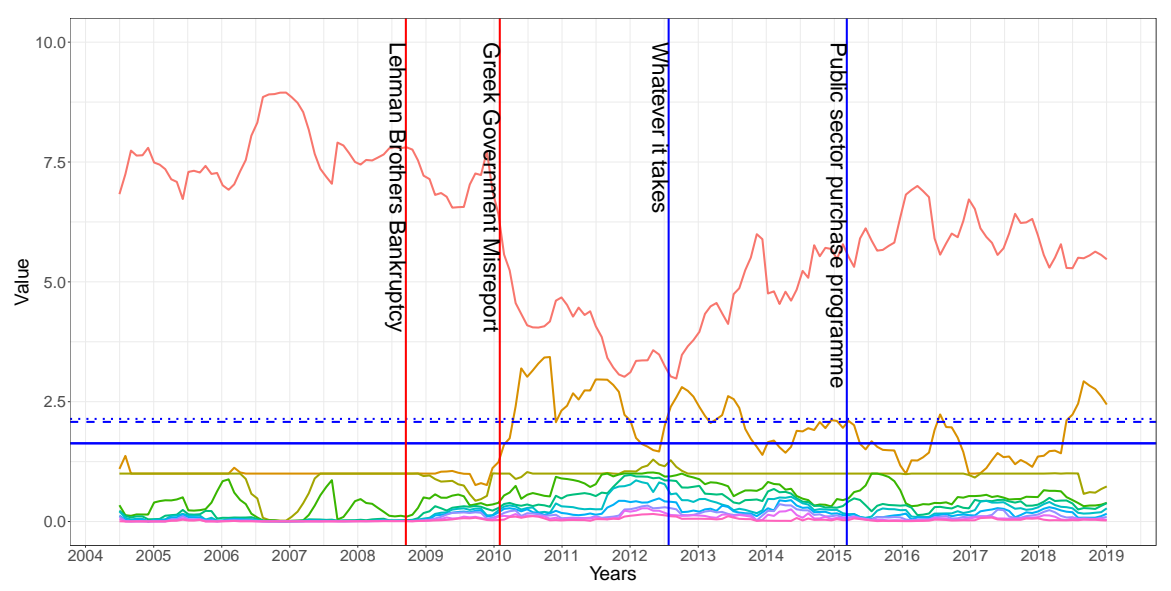

(b) Seven year yields

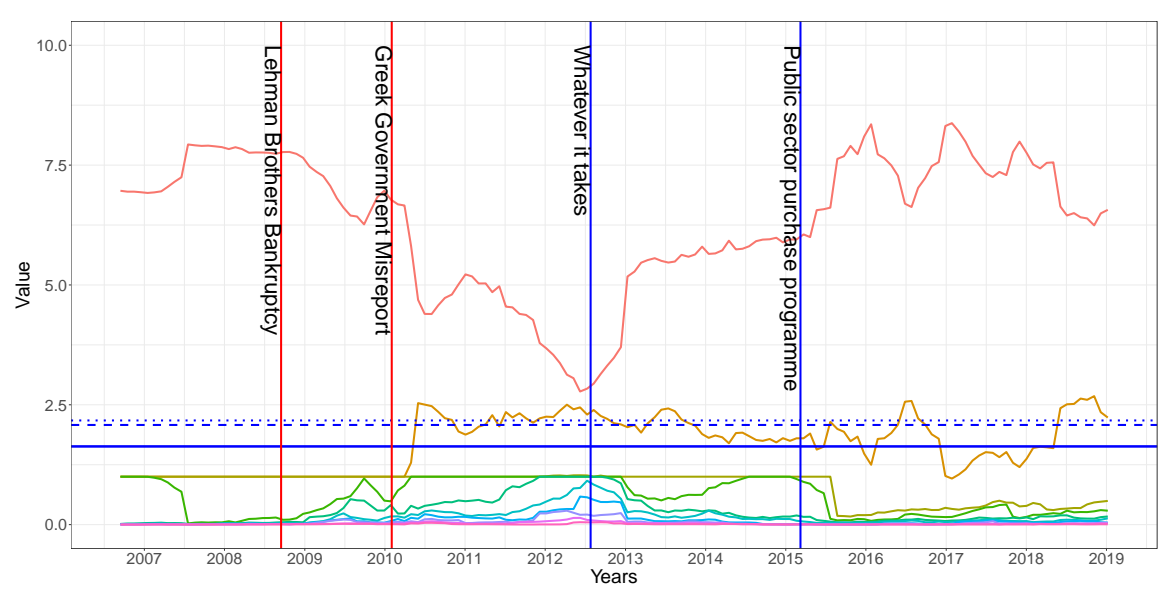

(c) Thirty year yields

Figure 10: Eigenvalue evolution for 3-month, 7-year, and 30-year yields.

Notes: the solid line indicates the Marčenko-Pastur theoretical bound, the dotted line indicates the simulated random model, and the thin dotted line indicates the rotational bound. For both the simulated random model and rotational random shuffling, 300 Monte Carlo simulations have been run. The dimension of the windows of the random matrix theory are 130 observations, step 22 observations.

rameters with apparently little interest. For instance, very large values of both the length and step parameters deliver a too aggregate and static image of synchronization, and they are not usefult to 
track synchronization through time. Accordingly, here we discuss only the results referring to alternatives with a plausible economic interpretation and selected according to a procedure minimizing the share of non significant correlation coefficients $e_{i, j}$ along all the windows.

\begin{tabular}{l|cccc}
\hline Maturity & Length & Step & $\begin{array}{c}\text { Average share of } \\
\text { non-significant coefficients }\end{array}$ & $\begin{array}{c}\text { Maximum share of } \\
\text { non-significant coefficients }\end{array}$ \\
\hline 1-year & 783 & 65 & 0.3401 & 0.7143 \\
1-year & 783 & 65 & 0.3401 & 0.7143 \\
5-years & 783 & 65 & 0.1640 & 0.4364 \\
5-years & 522 & 22 & 0.1591 & 0.4545 \\
10-years & 783 & 65 & 0.1070 & 0.4545 \\
10-years & 783 & 65 & 0.1071 & 0.4545 \\
\hline \hline
\end{tabular}

Table 2: Optimal combinations of parameters at the $1 \%$ level of significance.

Table 2 shows the combinations of the length and step parameters that minimize the average share of non-significant coefficients for the different maturities considered in our main analysis. The average percentage of non-significant coefficients at 5-years and 10-years yields is about $16 \%$ and $10 \%$ respectively. It increases to $30 \%$ for 1 -year yields. In addition, in most cases shown in Table 2 , the optimal length of the window is the largest possible given the size of our sample: 783 observations (i.e. windows of 3-year length). This is also a statistical artefact, since large windows have more observations and lead to higher levels of significance. The results of the synchronization analysis using the windows parameters in Table 2, confirm our main results (see Figure C.1 in Appendix C).

\subsection{Alternative filtering techniques}

It is well known that econometric results are very sensitive to the filter used to de-trend the data. For instance, the first differencing filter we use in our main analysis might generate spurious correlation (see e.g. Uhlig, 2009). We therefore carry out a sensitivity analysis of our main results also by taking second-order differences to eliminate residual spurious correlation. We then perform the PCA and RMT analysis and we find that the results are comparable to those obtained by using the first difference. ${ }^{20}$ Furthermore, Canova (1999); Baxter and King (1999) remarked that the first difference filter might overweight high frequency components in the time series. For this reason, we also perform a further battery of sensitivity analysis on data transformed by using the bandpass filter proposed in Christiano and Fitzgerald (2003). We employ this bandpass filter to select all the possible frequency

\footnotetext{
${ }^{20}$ For the sake of brevity, we do not report the results using second-order differences. However, they are available from the authors upon request.
} 
bands combining a lower bound comprised in the interval $\{2,22,65,130\}$ and an upper bound in the interval $\{22,65,130,261\}$, while keeping the dimension of the windows fixed. ${ }^{21}$ We repeat our synchronization analysis on all the foregoing frequency bands. Figure 11 shows the results about the resulting dominant eigenvalue, whereas Figure C.2 in Appendix C tracks the evolution of the second largest eigenvalue. The analysis of both principal components confirms he main results discussed in Section 5.2.

\subsection{Controlling for autocorrelation in the data}

Previous works using random matrix theory have shown that the empirical eigenvalue distribution becomes fat-tailed when time series are autocorrelated (see Aoyama et al., 2017). A fat-tailed distribution of eigenvalues implies that more principal components might turn significant according to the Marčenko-Pastur upper bound, even if these do not truly reflect a higher explained variance of the cross-correlations. To control for this problem, we perform a random rotational shuffling Monte Carlo exercise of the kind suggested by Aoyama et al. (2017) and Iyetomi et al. (2011). In particular we let:

$$
x_{n}\left(t_{i}\right) \rightarrow \begin{cases}x_{n}\left(t_{T-|i-\tau|}\right) & \text { if } i \leq \tau \\ x_{n}\left(t_{|i-\tau|}\right) & \text { if } i>\tau\end{cases}
$$

where $x_{n}\left(t_{i}\right)$ is the observation of the time series of the bond yields of the $n^{\text {th }}$ country at time $t_{i}$ with $i \in[1, T]$, and $\tau$ is a random number drawn from the interval $[1, T]$. The rotational random shuffling procedure divides the series in two parts: from 1 to $\tau$ and from $\tau+1$ to $T$. The two parts are then switched. The procedure is identical to a shift of the series by a random number, where the observations falling out of the length of the series are re-positioned at its beginning. Since every series is rotated by a different number, this rotation breaks the cross-correlation between the series, while maintaining the auto-correlation within the series (the observations of the series do not change order within the two parts). ${ }^{22}$ We perform multiple Monte Carlo iterations of the above rotational

\footnotetext{
${ }^{21}$ The window is kept as in the baseline analysis: six months window length (i.e 130 daily observations) and one month step length (i.e 22 daily observations). The combinations of lower and upper bounds are such that 2-22 selects only the frequencies between two days and one month, 2-65 between two days and one quarter, 65-261 between the quarter and the year, and so on for every possible combination.

${ }^{22}$ Consider, for instance, a time series of $T=130$ observations where we draw a random number $\tau=54$. The observations from 55 to 130 take the positions from the $|55-54|=1^{\text {st }}$ to the $|130-54|=76^{\text {th }}$. The observations from 1 to 54 take the positions from the $130-|1-54|=77^{\text {th }}$ to the $130-|54-54|=130^{\text {th }}$.
} 


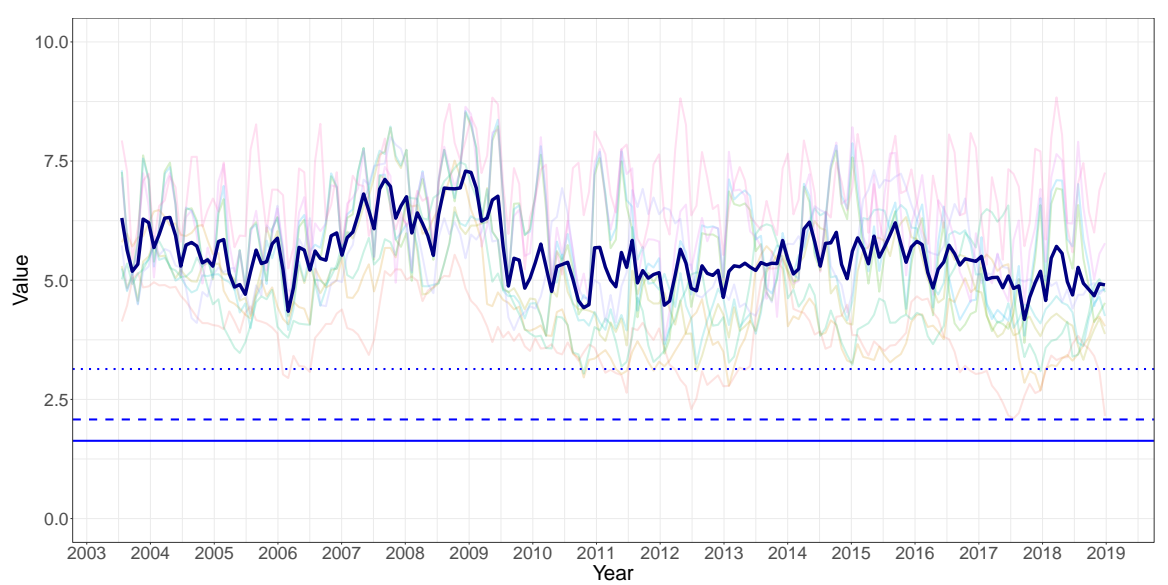

(a) One year yields

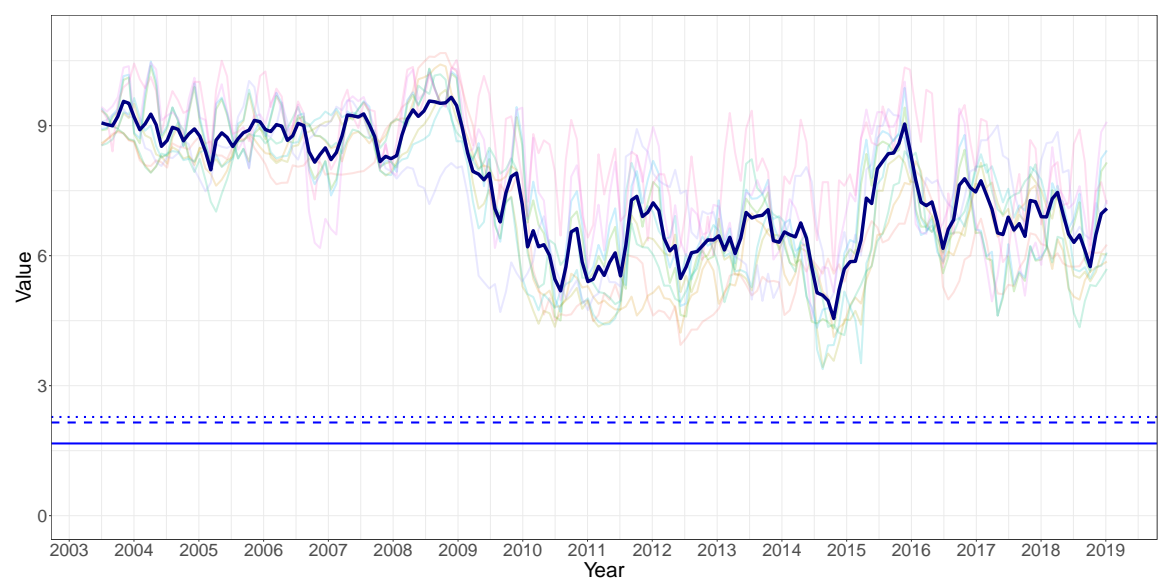

(b) Five year yields

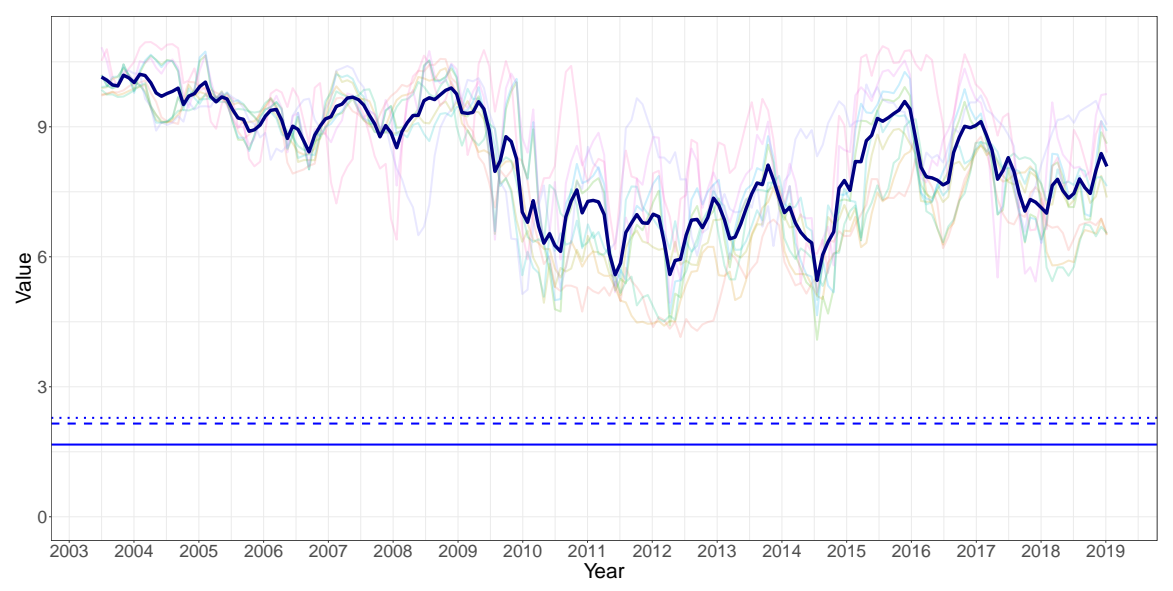

(c) Ten year yields

Figure 11: Evolution of the largest eigenvalue under different bandpass filters. The line in bold is the time average across the eigenvalues corresponding to the different frequency bands.

Notes: the solid line indicates the Marčenko-Pastur theoretical bound, the dotted line indicates the simulated random model, and the thin dotted line indicates the rotational bound. For both the simulated random model and rotational random shuffling, 300 Monte Carlo simulations have been run. The full dark blue line indicates the mean of the values given by the different frequencies selection. The selection of the frequencies is discussed in Section 6.2. 
shuffling exercise within each rolling window. For each window, we then compute the average of the maximum eigenvalues of the simulated series and the correspondent confidence intervals. The latter average constitutes a new upper bound that can be used to test the statistical significance of empirical eigenvalues. Eigenvalues lying above the random rotational shuffling bound contain a fraction of variance that is beyond the one explained by auto-correlation of the series and that it can then only be related to systematic cross-correlation. Notice that such an upper bound is larger, and thus more stringent, than the ones obtained through the procedure described in Section 4.

The upper bound stemming from above described procedure corresponds to the thin dotted lines in Figure 3 and in Figure 11. Clearly, all the components that were significant according to the standard RMT bounds, are also significant according tot the upper bounds based on rotational random shuffling. Our main results are thus robust to this additional restrictive test. In particular, the lack of synchronization for short term yields is even more evident, as the largest component becomes significant only in very few windows. In contrast, the largest component of medium- and long-term yields is always above the new bound and is thus always significant once we control for autocorrelation in the series. Finally, the significance of the second largest component is somehow reduced but not completely eliminated.

\subsection{Controlling for heavy tails in the data}

Besides the bias introduced by autocorrelation and discussed in the previous section, the presence of outliers in the filtered data might in general suggest that a Gaussian null model is not a correct benchmark for the selection of significant components. The work by Biroli et al. (2007) considers the case where the distribution of the observations is heavy-tailed. It also proposes a procedure to adapt the upper bounds of the Marčenko-Pastur law when the most extreme observation in a specific window overcomes a threshold that depends on the number of series and on their length. More precisely, let $S$ be the maximum standardized yield (i.e. the most extreme value) in a specific window of observations $X$. Then, assuming that the distribution of $X$ follows a heavy-tailed distribution, 
the RMT adjusted upper bound for the eigenvalues $\lambda_{\max }$ can be computed as follows:

$$
\lambda_{\max }= \begin{cases}\sigma^{2}\left(1+\sqrt{\frac{1}{Q}}\right)^{2}, & \text { if } S \leq(N T)^{\frac{1}{4}} \\ \left(\frac{1}{Q}+\frac{S^{2}}{T}\right)\left(1+\frac{T}{S^{2}}\right), & \text { if } S>(N T)^{\frac{1}{4}}\end{cases}
$$

where $Q=T / N$ and $\sigma^{2}=1$ because series are standardized. Figure 12 shows the results of our robustness check on 10-year yields using the procedure in Biroli et al. (2007). The new adjusted bound applies only for some windows in 2012. Even at this new level of significance, the first component remains strongly significant whereas the second one loses significance only in one window in 2012 . In the case of short-term yields the adjustment of the upper-bound applies for some windows during the period 2011-2012 as well as in 2016. The result is that the largest component becomes even less significant. In the case of 5-years yields, the new restriction applies for some windows in 2010, 2014, and 2017. The value of the new limit in some of the cases is significantly high, and it is mostly explained by the extreme values that some yields, especially Greek ones, reached during that periods. The periods of extreme values are nonetheless very limited and even the introduction of such high limits does not undermine the validity of our main results.

\section{Discussion and implications}

The evidence we presented so far robustly indicates that synchronization is not a statistically significant phenomenon for government bond yields in the Eurozone at short-term maturities. In contrast, yields at medium- and long-term maturities were very much synchronized until the arrival of the Great Recession and of the European Debt Crisis. The strains induced by these two crises generated an abrupt fall in medium- and long-term yields synchronization. This is highlighted by the loss in the share of the variance explained by the largest principal component and by the emergence of a second significant component during the period corresponding to the above two crises, i.e. between 2008 and 2014. Moreover, the elements of the eigenvectors associated with the above principal components were very heterogeneous during the periods corresponding to the two crises. Some of these eigenvector elements even took negative values during that period while other remained positive. Synchronization in medium- and long-term yields increased again since 2015, however without fully 


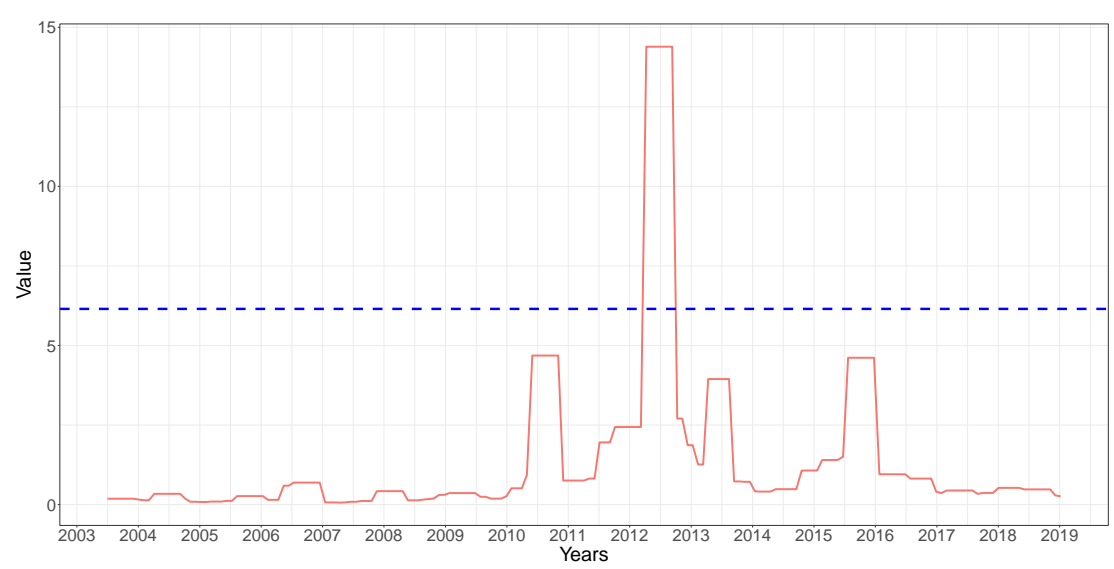

(a) Comparison between the $(N T)^{\frac{1}{4}}$ limit and the extreme observation $\mathrm{S}$. The upper bound of the random matrix theory should be adjusted only if the extreme value exceeds the bound.

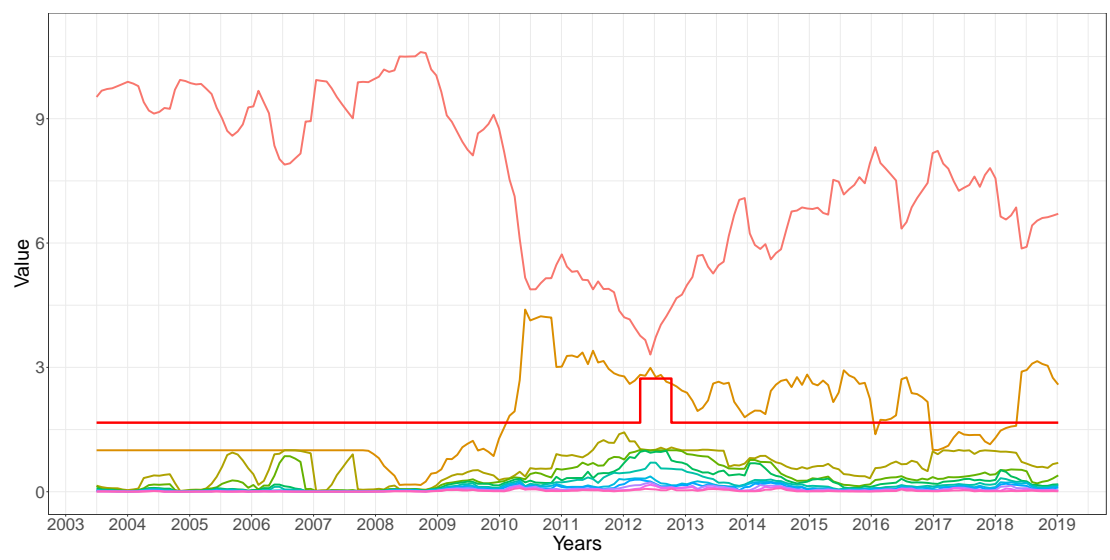

(b) Eigenvalues with the heavy-tailed adjusted upper bound.

Figure 12: Heavy-tails random null model for yields with 10-years maturity.

recovering of the high levels observable before the Great Recession.

We can discuss the above patterns by using the financial interpretation of principal components that we outlined in Section 4. In portfolio theory, the normalized elements of the eigenvector associated to significant eigenvalues represent the weights to be assigned to the different bonds in one portfolio, also called the eigenportfolio, with risk measured by the eigenvalue. In addition, positive and negative eigenvector components identify, respectively, long and short positions on the specific government bonds. Thus, if some differential between the eigenvector components exist, investors update their positions by reallocating their wealth over the bonds. This operations sustain the price of some bonds with respect to others. Speculation is feasible even if the components are of the same sign (see Avellaneda and Lee, 2010).

On these grounds, our results show the presence of divergence trades and flight-to-quality ef- 
fects that introduced asynchronicity in bond yields dynamics and that contributed to amplify the sovereign debt crisis in the Eurozone. More precisely, the fact that two eigenvalues were significant for bonds at 5 and 10 year maturities between 2008 and 2014 indicates that two eigenportfolios were likely to be traded in that period. Both portfolios were characterized by long position with respect to core Eurozone economies (Germany, France, Netherlands, Austria, Finland and Belgium) and low exposition or even short positions with respect to the peripheral economies (Spain, Greece, Portugal, Italy and Ireland). Moreover, at the peak of European Debt Crisis (i.e. between 2011 and 2013) the eigenportfolio with lower risk, i.e. the one associated with the second largest eigenvalue, implied short positions on government bonds of all Eurozone countries but Germany, Netherlands and Finland. Accordingly, in those periods government bonds of those countries became a safe asset (see Caballero et al., 2017) over which strong long positions were taken.

The above documented dynamics of bond yields has several implications for the conduct of policy in the Eurozone. As a matter of fact, yields asynchronicity hampers the effectiveness of the common monetary policy via the interest rate channel. In particular, finding that eigenvector components of government bond principal components are heterogeneous and with differing signs, indicates that common monetary policy actions by the European central bank affect asset returns in different fashion across different countries. For interest rate policies to be effective in the Eurozone, instead, government bond yields of the member states should move together, as if they were a unique interest rate. Furthermore, government bonds are the main type of collateral used to secure loans in interbank markets and their value directly impact on the ability of banks to collect liquidity. In that respect, the Eurozone differs from other monetary unions, as several bonds are associated with the same currency. As a result, if the dynamics of the interest rates on government bonds differ, liquidity is likely to move heterogeneously across Eurozone countries. Last but not least, excessive divergence in government bond yields may map into divergent dynamics of government debts within a monetary union, complicating fiscal and monetary policy interactions in response to economic shocks and even posing threats to the existence of the union itself.

The importance of government bond yields synchronicity for the functioning of Eurozone monetary policy has also been acknowledged by members of the ECB board in the past (see e.g. Draghi, 2012; Cœuré, 2017). In addition, the presence of excessive divergence in sovereign bond premia 
has been used to support the implementation of unconventional monetary policies in the Eurozone. With this perspective in mind, Figure 3 discussed in Section 5.2 reveals that the turning point in bond yields synchronization coincides with the notorious "whatever it takes" statement by the ECB President Mario Draghi on $26^{\text {th }}$ July 2012. Synchronization gained momentum after the announcement, as the variance explained by the first component (and measured by the value of the dominant eigenvalue) rapidly increased, while the importance of the second principal component diminished. These trends continued also after the start of the Public Security Purchase Program (PSPP), explicitly targeting the purchase of Eurozone government bonds in the secondary market (cf. Figure 3). Nevertheless, the second component remained significant in many windows also after the start of the PSPP, with eigenvector elements of some countries still being negative (see Figures 8 and 9 as well as Section 5.2). This suggests that divergence trades survived after the implementation of the latter program. Clearly, the foregoing case study does not allow one to infer any causal impact of unconventional monetary policies. Nonetheless, it suggests the presence of a relation between the chronology of QE events and a significant recovery of bond yields synchronization in the Eurozone. Moreover, the fact that the statistical significance of a second common factor persists also after bond purchasing programme poses concerns about a possible return of diverging yields dynamics once QE programs will be over.

\section{Concluding remarks}

We have employed a novel econometric procedure combining principal component analysis and random matrix theory to detect significant synchronization in the daily time series of Eurozone government bond yields at different maturities. We found that bond yields at short maturities are in general poorly synchronized. In contrast, bond yields at medium and long term maturities were highly synchronized until the onset of the Great Recession. This recession and the subsequent European Debt Crisis resulted in a disruption of synchronization in the government bond yields of Eurozone countries. This is evidenced by the loss in significance of the first principal component and by the emergence of a significant second component. In addition, we find that in the aforementioned periods the eigenvector elements associated with significant components became highly heterogeneous, and even displayed opposite signs across countries. We also provided a financial in- 
terpretation of our main results grounded on eigenportfolio theory (Avellaneda and Lee, 2010) and we discussed how our evidence indicates that yields asynchronicity can be related to the presence of divergence trades and flight-to-quality effects. Finally, we showed that our results are robust to the use of different maturities, the use of alternative windows to compute principal components, the use of different filtering techniques, the presence of autocorrelation and heavy-tails in the data.

Our study could be extended in several directions. First of all, we did not consider lagged correlations. Such an extension is possible either by implementing additional static factor models (Bouchaud and Potters, 2015) or by focusing directly on dynamic factor models. This second extension would allow one to precisely evaluate the pass-through of monetary policy across different yield maturities. Secondly, our study could be extended to encompass the main refinancing operation rate (set by the central bank) and different banks rates (the interbank market one as well as the ones of loans). An enlarged dataset might also include data on real economic variables as well as monetary ones in order to investigate synchronization between financial and real activities.

\section{Acknowledgements}

We thank Giorgio Fagiolo, Paolo Barucca, Davide Fiaschi, Simone Alfarano, Anindya Chakrabarti, and Marcello Minenna for insightful comments and discussions. We are also grateful to Roberto Sichera and Pietro Pizzuto for their help with the "ConvergenceClubs" R package, available at the CRAN archive. We also thank participants to the WEHIA 2019 conference, City University of London, UK, to the CFE-CMStatistics 2020, Virtual Conference and to the Como lake Summer School 2019, Como, Italy and to various seminars at Scuola Superiore Sant'Anna and the University of Côte d'Azur, where earlier versions of this paper were presented. Finally, this work owes much to the support of Tarek Amyuni and Skema Business School, who granted us access to the Bloomberg dataset. 


\section{References}

Aoyama, H., Y. Fujiwara, and W. Souma (2017). Macro-Econophysics: New Studies on Economic Networks and Synchronization. Physics of Society: Econophysics and Sociophysics. Cambridge: Cambridge University Press.

Arouri, M., F. Jawadi, and D. K. Nguyen (2013). What can we tell about monetary policy synchronization and interdependence over the 2007-2009 global financial crisis? Fournal of Macroeconomics $36,175-187$.

Avellaneda, M. and J. H. Lee (2010). Statistical arbitrage in the US equities market. Quantitative Finance 10(7), 761-782.

Bai, J. and S. Ng (2002). Determining the number of factors in approximate factor models. Econometrica 70(1), 191-221.

Bai, J. and S. Ng (2008). Large dimensional factor analysis. Foundations and Trends in Econometrics 3(2), 89-163.

Baxter, M. and R. G. King (1999). Measuring Business Cycles: Approximate Band-Pass Filters For Economic Time Series. The Review of Economics and Statistics 81(4), 575-593.

Belo, F. (2001). Some Facts About the Cyclical Convergence in the Euro Zone. Working Papers, Banco de Portugal - Economics and Research Department (w200107).

Billio, M., M. Getmansky, A. W. Lo, and L. Pelizzon (2012). Econometric measures of connectedness and systemic risk in the finance and insurance sectors. Fournal of financial economics 104(3), 535-559.

Biroli, G., J.-P. Bouchaud, and M. Potters (2007). Extreme value problems in random matrix theory and other disordered systems. Journal of Statistical Mechanics: Theory and Experiment (P07019).

Blanchard, O. (2016). The phillips curve: Back to the '60s? American Economic Review 106(5), 31-34.

Bouchaud, J. P. and M. Potters (2015). Financial applications of random matrix theory: a short review. In G. Akemann, J. Baik, and P. D. Francesco (Eds.), The Oxford Handbook of Random Matrix Theory. (Online edition) Oxford: Oxford University Press.

Caballero, R. J., E. Farhi, and P.-O. Gourinchas (2017). The safe assets shortage conundrum. fournal of Economic Perspectives 31(3), 29-46.

Canova, F. (1999). Does detrending matter for the determination of the reference cycle and the selection of turning points? The Economic fournal 109(452), 126-150.

Christiano, L. and T. Fitzgerald (2003). The band pass filter. International Economic Review 44(2), 435-465.

Christiano, L. J., M. Eichenbaum, and C. L. Evans (1999). Monetary policy shocks: What have we learned and to what end? In J. B. Taylor and M. Woodford (Eds.), Handbook of Macroeconomics, Handbook of Macroeconomics, pp. 65-148. Elsevier.

Cochrane, J. H. and M. Piazzesi (2005). Bond Risk Premia. American Economic Review 95(1), 138-160. 
Cœuré, B. (2017). Speech at the workshop "Monetary policy in non-standard times". (Frankfurt am Main, 11 September 2017).

Coibion, O. and Y. Gorodnichenko (2015). Is the phillips curve alive and well after all? inflation expectations and the missing disinflation. American Economic fournal: Macroeconomics 7(1), 197232.

Diebold, F. X. and K. Yllmaz (2014). On the network topology of variance decompositions: Measuring the connectedness of financial firms. Fournal of Econometrics 182(1), 119 - 134.

Dow, S., A. Montagnoli, and O. Napolitano (2012). Interest Rates and Convergence across Italian Regions. Regional Studies 46(7), 893-905.

Draghi, M. (2012). Speech by Mario Draghi, President of the European Central Bank at the Global Investment Conference in London, 26 July 2012. (London, 26 July 2012).

Geweke, J. (1982). Measurement of linear dependence and feedback between multiple time series. Journal of the American Statistical Association 77(378), 304-313.

Guerini, M., D. Luu, and M. Napoletano (2019). Synchronization patterns in the european union. GREDEG Working Papers (30).

Guo, D., P. P. Boyle, C. Weng, and T. S. Wirjanto (2018). Eigen Portfolio Selection: A Robust Approach to Sharpe Ratio Maximization. SSRN Electronic fournal: available at SSRN: https://ssrn.com/abstract=3070416 or http://dx.doi.org/10.2139/ssrn.3070416.

Iyetomi, H., Y. Nakayama, H. Aoyama, Y. Fujiwara, Y. Ikeda, and W. Souma (2011). Fluctuationdissipation theory of input-output interindustrial correlations. Physical Review E 83(016103).

Kapetanios, G. (2010). A testing procedure for determining the number of factors in approximate factor models with large datasets. Journal of Business and Economic Statistics 28(3), 397-409.

Kritzman, M., Y. Li, S. Page, and R. Rigobon (2011). Principal components as a measure of systemic risk. The Journal of Portfolio Management 37(4), 112-126.

Laloux, L., P. Cizeau, J.-P. Bouchaud, and M. Potters (1999, Aug). Noise dressing of financial correlation matrices. Phys. Rev. Lett. 83(7), 1467-1470.

Laloux, L., P. Cizeau, M. Potters, and J.-P. Bouchaud (2000). Random matrix theory and financial correlations. International fournal of Theoretical and Applied Finance 03(03), 391-397.

Lettau, M. and A. Madhavan (2018). Exchange-traded funds 101 for economists. Fournal of Economic Perspectives 32(1), 135-54.

Ludvigson, S. C. and S. Ng (2007). The empirical risk-return relation: A factor analysis approach. fournal of Financial Economics 83(1), 171-222.

Ludvigson, S. C. and S. Ng (2010). A Factor Analysis of Bond Risk Premia. In A. Ullah and D. E. A. Giles (Eds.), Adjustment and growth in European Monetary Union. (Online edition) New York: Chapman and Hall/CRC.

Manski, C. F. (1993). Identification of endogenous social effects: The reflection problem. The review of economic studies 60(3), 531-542. 
Marčenko, V. A. and L. A. Pastur (1967). Distribution of eigenvalues for some sets of random matrices. Mathematics of the USSR-Sbornik 1(4), 457-483.

Münnix, M. C., T. Shimada, R. Schäfer, F. Leyvraz, T. H. Seligman, T. Guhr, and H. E. Stanley (2012). Identifying states of a financial market. Scientific reports 2(1), 1-6.

Onatski, A. (2009). Testing hypotheses about the number of factors in large factor models. Econometrica 77(5), 1447-1479.

Onatski, A. (2010). Determining the number of factors from empirical distribution of eigenvalues. The Review of Economics and Statistics 92(4), 1004-1016.

Phillips, P. C. B. and D. Sul (2007). Transition modeling and econometric convergence tests. Econometrica 75(6), 1771-1855.

Reis, R. and M. W. Watson (2010). Relative goods' prices, pure inflation, and the phillips correlation. American Economic fournal: Macroeconomics 2(3), 128-57.

Sichera, R. and P. Pizzuto (2019). ConvergenceClubs: A Package for Performing the Phillips and Sul's Club Convergence Clustering Procedure. The R Journal.

Stock, J. H. and M. W. Watson (2002). Forecasting Using Principal Components From a Large Number of Predictors. Fournal of the American Statistical Association 97(460), 1167 - 1179.

Tumminello, M., F. Lillo, and R. N. Mantegna (2010). Correlation, hierarchies, and networks in financial markets. Fournal of Economic Behavior \& Organization 75(1), 40-58.

Uhlig, H. (2009). Comment on "How Has the Euro Changed the Monetary Transmission Mechanism?". In NBER Macroeconomics Annual 2008, Volume 23, pp. 141-152. National Bureau of Economic Research, Inc.

Vajanne, L. (2007). Integration in euro area retail banking markets : convergence of credit interest rates. Bank of Finland Research Discussion Papers, $n$. 27/2007.

Wälti, S. (2011). Stock market synchronization and monetary integration. fournal of International Money and Finance 30(1), 96-110. 


\section{Appendix A. Dataset}

\section{A.1 Description of the time series}

\begin{tabular}{lllll}
\hline Bloomberg ID & Maturity & Country & Begin & End \\
\hline GTBEF3M Govt & 3 months & BE & $2006-07-13$ & $2019-02-19$ \\
GTFRF3M Govt & 3 months & FR & $2006-07-13$ & $2019-02-19$ \\
GTDEM3M Govt & 3 months & DE & $2006-07-13$ & $2019-02-19$ \\
GTGRD3M Govt & 3 months & GR & $2007-10-22$ & $2019-02-19$ \\
GTITL3M Govt & 3 months & IT & $2006-07-13$ & $2019-02-19$ \\
GTNLG3M Govt & 3 months & NE & $2006-07-13$ & $2019-02-19$ \\
GTPTE3M Govt & 3 months & PT & $2011-08-08$ & $2019-02-19$ \\
GTESP3M Govt & 3 months & SP & $2006-07-13$ & $2019-02-19$ \\
GTBEF6M Govt & 6 months & BE & $2006-07-13$ & $2019-02-18$ \\
GTFRF6M Govt & 6 months & FR & $2006-07-13$ & $2019-02-18$ \\
GTDEM6M Govt & 6 months & DE & $2006-07-13$ & $2019-02-18$ \\
GTGRD6M Govt & 6 months & GR & $2007-10-22$ & $2019-02-18$ \\
GTITL6M Govt & 6 months & IT & $2006-07-13$ & $2019-02-18$ \\
GTNLG6M Govt & 6 months & NE & $2006-07-13$ & $2019-02-18$ \\
GTPTE6M Govt & 6 months & PT & $2011-08-08$ & $2019-02-18$ \\
GTESP6M Govt & 6 months & SP & $2006-07-13$ & $2019-02-18$ \\
GTATS1Y Govt & 1 year & AT & $2008-04-03$ & $2019-02-21$ \\
GTBEF1Y Govt & 1 year & BE & $2003-01-20$ & $2019-02-21$ \\
GTFIM1Y Govt & 1 year & FI & $2003-01-20$ & $2019-02-21$ \\
GTFRF1Y Govt & 1 year & FR & $2003-01-20$ & $2019-02-21$ \\
GTDEM1Y Govt & 1 year & DE & $2003-01-20$ & $2019-02-21$ \\
GTGRD1Y Govt & 1 year & GR & $2007-03-02$ & $2019-02-21$ \\
GTIEP1Y Govt & 1 year & IR & $2003-01-20$ & $2019-02-21$ \\
GTITL1Y Govt & 1 year & IT & $2003-01-20$ & $2019-02-21$ \\
GTPTE1Y Govt & 1 year & PT & $2003-01-20$ & $2019-02-21$ \\
GTESP1Y Govt & 1 year & SP & $2011-08-08$ & $2019-02-21$ \\
\hline \hline
\end{tabular}

Table A.1: Short term daily data. Source: Bloomberg. Countries: AT = Austria, BE = Belgium, FI = Finland, FR = France, $\mathrm{DE}=$ Germany, GR = Greece, IR = Ireland, IT = Italy, NE = Netherlands, Pt = Portugal, SP = Spain . 


\begin{tabular}{|c|c|c|c|c|}
\hline Bloomberg ID & Maturity & Country & Begin & End \\
\hline GTATS2Y Govt & 2 years & AT & 1999-11-02 & 2019-03-06 \\
\hline GTBEF2Y Govt & 2 years & $\mathrm{BE}$ & 1999-11-02 & 2019-03-06 \\
\hline GTFIM2Y Govt & 2 years & FI & 1999-11-02 & 2019-03-06 \\
\hline GTFRF2Y Govt & 2 years & FR & 1999-11-02 & 2019-03-06 \\
\hline GTDEM2Y Govt & 2 years & $\mathrm{DE}$ & 1999-11-02 & 2019-03-06 \\
\hline GTITL2Y Govt & 2 years & IT & 1999-11-02 & 2019-03-06 \\
\hline GTPTE2Y Govt & 2 years & $\mathrm{PT}$ & 1999-11-02 & 2019-03-06 \\
\hline GTESP2Y Govt & 2 years & SP & 1999-11-02 & 2019-03-06 \\
\hline GTATS3Y Govt & 3 years & AT & 1999-11-02 & 2019-02-21 \\
\hline GTBEF3Y Govt & 3 years & $\mathrm{BE}$ & 1999-11-02 & 2019-02-21 \\
\hline GTFIM3Y Govt & 3 years & FI & 1999-11-02 & 2019-02-21 \\
\hline GTFRF3Y Govt & 3 years & FR & 1999-11-02 & 2019-02-21 \\
\hline GTDEM3Y Govt & 3 years & $\mathrm{DE}$ & 1999-11-02 & 2019-02-21 \\
\hline GTGRD3Y Govt & 3 years & GR & 2007-03-01 & 2019-02-21 \\
\hline GTIEP3Y Govt & 3 years & IR & 1999-11-02 & 2019-02-21 \\
\hline GTNLG3Y Govt & 3 years & $\mathrm{NE}$ & 1999-11-02 & 2019-02-21 \\
\hline GTPTE3Y Govt & 3 years & $\mathrm{PT}$ & 1999-11-02 & 2019-02-21 \\
\hline GTESP3Y Govt & 3 years & SP & 1999-11-02 & 2019-02-21 \\
\hline GTATS5Y Govt & 5 years & AT & 1999-11-02 & 2019-02-21 \\
\hline GTBEF5Y Govt & 5 years & $\mathrm{BE}$ & 1999-11-02 & $2019-02-21$ \\
\hline GTFIM5Y Govt & 5 years & FI & 2007-10-12 & $2019-02-21$ \\
\hline GTFRF5Y Govt & 5 years & FR & 1999-11-02 & $2019-02-21$ \\
\hline GTDEM5Y Govt & 5 years & $\mathrm{DE}$ & 1999-11-02 & $2019-02-21$ \\
\hline GTGRD5Y Govt & 5 years & GR & 2007-03-02 & 2019-02-21 \\
\hline GTIEP5Y Govt & 5 years & IR & 1999-11-02 & $2019-02-21$ \\
\hline GTITL5Y Govt & 5 years & IT & 1999-11-02 & $2019-02-21$ \\
\hline GTNLG5Y Govt & 5 years & $\mathrm{NE}$ & 1999-11-02 & $2019-02-21$ \\
\hline GTPTE5Y Govt & 5 years & $\mathrm{PT}$ & 1999-11-02 & $2019-02-21$ \\
\hline GTESP5Y Govt & 5 years & SP & 1999-11-02 & 2019-02-21 \\
\hline GTATS7Y Govt & 7 years & AT & 2003-11-06 & 2019-03-06 \\
\hline GTBEF7Y Govt & 7 years & $\mathrm{BE}$ & 2003-11-06 & 2019-03-06 \\
\hline GTFIM7Y Govt & 7 years & FI & 2003-11-06 & 2019-03-06 \\
\hline GTFRF7Y Govt & 7 years & FR & 2003-11-06 & 2019-03-06 \\
\hline GTDEM7Y Govt & 7 years & $\mathrm{DE}$ & 2003-11-06 & 2019-03-06 \\
\hline GTGRD7Y Govt & 7 years & GR & 2007-03-02 & 2019-03-06 \\
\hline GTIEP7Y Govt & 7 years & IR & 2003-11-06 & 2019-03-06 \\
\hline GTITL7Y Govt & 7 years & IT & 2003-11-06 & 2019-03-06 \\
\hline GTPTE7Y Govt & 7 years & $\mathrm{PT}$ & 2003-11-06 & 2019-03-06 \\
\hline GTESP7Y Govt & 7 years & SP & 2003-11-06 & 2019-03-06 \\
\hline
\end{tabular}

Table A.2: Medium term daily data. Source: Bloomberg. Countries: AT = Austria, BE = Belgium, FI = Finland, FR = France, $\mathrm{DE}=$ Germany, GR = Greece, $\mathrm{IR}=$ Ireland, $\mathrm{IT}=$ Italy, NE = Netherlands, $\mathrm{Pt}=$ Portugal, $\mathrm{SP}=\mathrm{Spain}$. 


\begin{tabular}{|c|c|c|c|c|}
\hline Bloomberg ID & Maturity & Country & Begin & End \\
\hline GTATS10Y Govt & 10 years & AT & 1999-11-02 & 2019-02-18 \\
\hline GTBEF10Y Govt & 10 years & $\mathrm{BE}$ & 1999-11-02 & $2019-02-18$ \\
\hline GTFIM10Y Govt & 10 years & FI & 1999-11-02 & $2019-02-18$ \\
\hline GTFRF10Y Govt & 10 years & FR & 1999-11-02 & $2019-02-18$ \\
\hline GTDEM10Y Govt & 10 years & $\mathrm{DE}$ & 1999-11-02 & $2019-02-18$ \\
\hline GTGRD10Y Govt & 10 years & GR & 2007-03-02 & $2019-02-18$ \\
\hline GTIEP10Y Govt & 10 years & IR & 1999-11-02 & $2019-02-18$ \\
\hline GTITL10Y Govt & 10 years & IT & 1999-11-02 & 2019-02-18 \\
\hline GTNLG10Y Govt & 10 years & $\mathrm{NE}$ & 1999-11-02 & $2019-02-18$ \\
\hline GTPTE10Y Govt & 10 years & $\mathrm{PT}$ & 1999-11-02 & $2019-02-18$ \\
\hline GTESP10Y Govt & 10 years & SP & 1999-11-02 & $2019-02-18$ \\
\hline GTATS15Y Govt & 15 years & AT & $2002-10-18$ & 2019-03-06 \\
\hline GTBEF15Y Govt & 15 years & $\mathrm{BE}$ & $2002-10-18$ & 2019-03-06 \\
\hline GTFIM15Y Govt & 15 years & FI & $2002-10-18$ & 2019-03-06 \\
\hline GTFRF15Y Govt & 15 years & FR & $2002-10-18$ & 2019-03-06 \\
\hline GTDEM15Y Govt & 15 years & $\mathrm{DE}$ & $2002-10-18$ & 2019-03-06 \\
\hline GTGRD15Y Govt & 15 years & GR & 2007-03-02 & 2019-03-06 \\
\hline GTIEP15Y Govt & 15 years & IR & $2002-10-18$ & 2019-03-06 \\
\hline GTITL15Y Govt & 15 years & IT & $2002-10-18$ & 2019-03-06 \\
\hline GTNLG15Y Govt & 15 years & $\mathrm{NE}$ & $2002-10-18$ & 2019-03-06 \\
\hline GTPTE15Y Govt & 15 years & $\mathrm{PT}$ & $2002-10-18$ & 2019-03-06 \\
\hline GTESP15Y Govt & 15 years & SP & $2002-10-18$ & 2019-03-06 \\
\hline GTATS20Y Govt & 20 years & AT & 2005-05-19 & 2019-03-06 \\
\hline GTBEF20Y Govt & 20 years & $\mathrm{BE}$ & 2005-05-19 & 2019-03-06 \\
\hline GTFRF20Y Govt & 20 years & FR & 2005-05-19 & 2019-03-06 \\
\hline GTDEM20Y Govt & 20 years & $\mathrm{DE}$ & 2005-05-19 & 2019-03-06 \\
\hline GTGRD20Y Govt & 20 years & GR & 2012-03-13 & 2019-03-06 \\
\hline GTIEP20Y Govt & 20 years & IR & 2005-05-19 & 2019-03-06 \\
\hline GTITL20Y Govt & 20 years & IT & 2005-05-19 & 2019-03-06 \\
\hline GTPTE20Y Govt & 20 years & PT & 2014-01-17 & 2019-03-06 \\
\hline GTESP20Y Govt & 20 years & SP & $2005-05-20$ & 2019-03-06 \\
\hline GTATS30Y Govt & 30 years & AT & $2006-03-16$ & 2019-03-06 \\
\hline GTBEF30Y Govt & 30 years & $\mathrm{BE}$ & $2006-03-16$ & 2019-03-06 \\
\hline GTFIM30Y Govt & 30 years & FR & 2012-06-29 & 2019-03-06 \\
\hline GTFRF30Y Govt & 30 years & $\mathrm{DE}$ & $2006-03-16$ & 2019-03-06 \\
\hline GTDEM30Y Govt & 30 years & GR & $2006-03-16$ & 2019-03-06 \\
\hline GTIEP30Y Govt & 30 years & IR & $2015-02-04$ & 2019-03-06 \\
\hline GTITL30Y Govt & 30 years & IT & $2006-03-16$ & 2019-03-06 \\
\hline GTNLG30Y Govt & 30 years & $\mathrm{NE}$ & $2006-03-16$ & 2019-03-06 \\
\hline GTPTE30Y Govt & 30 years & $\mathrm{PT}$ & 2006-03-16 & 2019-03-06 \\
\hline GTESP30Y Govt & 30 years & SP & $2006-03-16$ & 2019-03-06 \\
\hline
\end{tabular}

Table A.3: Long term daily data. Source: Bloomberg. Countries: AT = Austria, BE = Belgium, FI = Finland, FR = France, $\mathrm{DE}=$ Germany, GR = Greece, $\mathrm{IR}=$ Ireland, $\mathrm{IT}=$ Italy, NE = Netherlands, $\mathrm{Pt}=$ Portugal, $\mathrm{SP}=$ Spain . 


\section{Appendix B. Factor analysis}

Our main procedure based on the detection of statistically significant principal components has strong relations with static factor models (Stock and Watson, 2002; Onatski, 2010). The reason is that static factors are typically estimated by using principal component analysis. In this appendix we complement our main analysis by estimating factors and by tracking their evolution over time. More precisely, a static factor model decomposes each time series in a component driven by few common factors and an idiosyncratic component (see e.g. Stock and Watson, 2002):

$$
X_{t}=B F_{t}+e_{t}
$$

where $\underset{N \times T}{X}$ is the matrix of the time series, $\underset{N \times N}{B}$ is the matrix of the loadings of each factor, $\underset{N \times T}{F}$ is the matrix of the factors, and $\underset{1 \times T}{e}$ is the vector of the idiosyncratic components.

The matrices $B$ and $F$ are estimated via the PCA described in section 4.1 above. The covariance matrix $\Gamma_{Y}$ of the $n$ time series $Y$ can be factorized as

$$
\Gamma_{Y}=E\left[X_{T} X_{T}^{\prime}\right]=U \Lambda U^{T}
$$

where $\Lambda_{N \times N}$ is the matrix with the ordered eigenvalues of the covariance matrix on the diagonal, while $\underset{N \times N}{U}$ is the orthogonal matrix of the corresponding eigenvectors. Once the first $K$ eigenvalues and eigenvectors are selected via the random matrix procedure illustrated in Section 4.2, the factor model boils down to:

$$
X_{t}=\tilde{B} \tilde{F}_{t}+e_{t}
$$

where $\tilde{B}$ has dimension $N \times K$ and $\tilde{F}$ has dimension $K \times T$. If we define the factor loadings ${ }^{23}$ as $\tilde{B}=\sqrt{n} \tilde{U}$, then the matrix $\tilde{F}$ can be estimated by OLS such that:

$$
\tilde{F}=\left(\tilde{B}^{T} \tilde{B}\right)^{-1} \tilde{B}^{T} X
$$

Lastly, factors can be identified up to a rotation

$$
\hat{F}=\Theta \tilde{F}
$$

where $\Theta=\left[\begin{array}{cc}\cos (\theta) & \sin (\theta) \\ -\sin (\theta) & \cos (\theta)\end{array}\right]$ is the rotation matrix. Consider for instance the two models

$$
\begin{gathered}
X_{(1)}=\tilde{B} \tilde{F}+e \\
X_{(2)}=\tilde{B} \Theta \tilde{F}+e
\end{gathered}
$$

Assuming $E(e)=0$ and computing the covariance matrices we can show that $\Gamma_{X_{(2)}}=E\left[X_{T} X_{T}^{\prime}\right]=$ $(\tilde{B} \Theta \tilde{F}+e)^{\prime}(\tilde{B} \Theta \tilde{F}+e)=\tilde{F}^{\prime} \Theta^{\prime} \tilde{B}^{\prime} \tilde{B} \Theta \tilde{F}+e^{\prime} e$. Using $\tilde{B}=\tilde{U}$, we have that $\tilde{B}^{\prime} \tilde{B}=I$, while $\Theta^{\prime} \Theta=I$ by definition of the rotation matrix. We thus have $\Gamma_{X_{(2)}}=\tilde{F}^{\prime} \tilde{F}+e^{\prime} e=\Gamma_{X_{(1)}}$.

For each window we estimate the first two factors according to our selection procedure. More precisely, for each window, we compute the Spearman correlation of the factors with the filtered time series. Given the large number of windows (around 200), we report the correlation coefficients aggregated by year in Table B.1 and B.2 for 1-year yields, in Table B.3 and B.4 for 5-year yields, and

\footnotetext{
${ }^{23}$ This choice of loadings is common in the literature for its asymptotic properties (See e.g. Stock and Watson 2002). In our case, however, given $n=11$ we cannot claim any particular asymptotic property, so that the multiplication by $\sqrt{n}$ can be seen as a simple rescaling factor.
} 
in Table B.5, B.6 for 10-year yields. An inspection of the tables shows that the correlations of the peripheral countries tend to be weaker with the first factor in times of crisis and stronger in general with the second factor. To help a visual inspection, Figure B.1 shows the factors on the graph in levels for 5 and 10 year yields. ${ }^{24}$

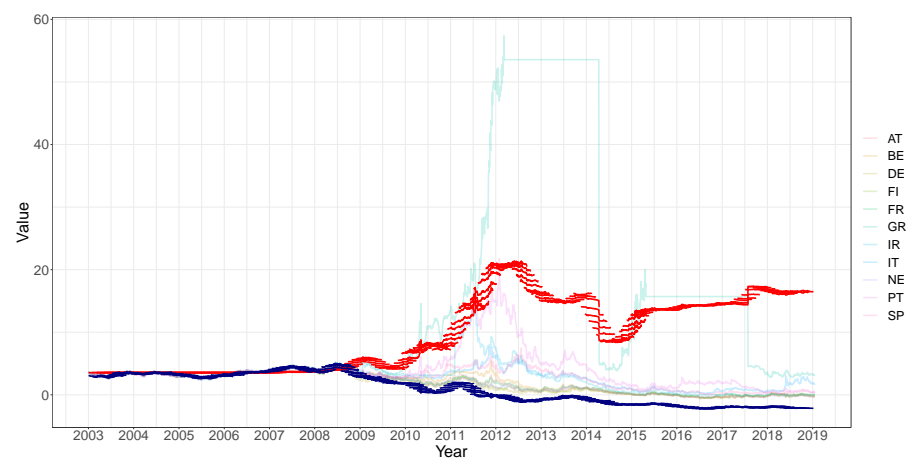

(a) 5-year yields

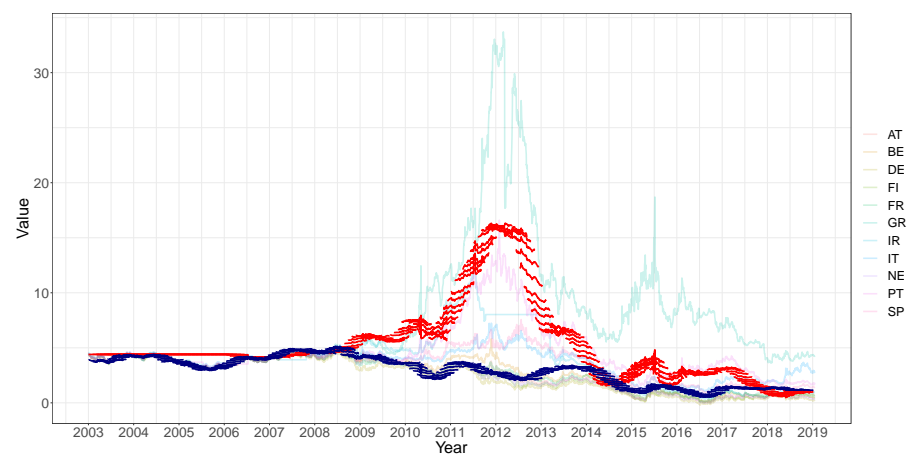

(b) 10-year yields

Figure B.1: Factors evolution.

Notes: the first factor is in blue, while the second factor is in red.

The analysis of the factors supports the conclusions reached in Section 5.2 that bond yields at 1-year maturity are poorly synchronized. In contrast, one factor has been driving all the series of 5- and 10-years bonds up to 2008. From 2008 on some series are still strongly correlated with the factor, while other lose correlation. In some cases, such as year 2011 and 2012, we have that the correlations even differ in sign between the two groups of countries. Notice that this dynamic and the signs of the correlations are robust for all the three maturities and for both filtered and raw data. The analysis also supports the interpretation of the second factor, which from year 2008 on robustly shows a higher correlation with the peripheral countries and generally a low correlation with the core countries.

\footnotetext{
${ }^{24}$ Unfortunately the 1 year yields does not allow a friendly visualization due to the extreme values of some yields, especially Greece. The high frequency of the data also makes a visualization of the filtered time series and their factors useless. Finally, notice that already for the 5 year yields, the levels of the second factor are already influenced by the extreme values of some time series. It is worth remembering, however, that factors for the time series in levels are derived from the factors computed on the first differences by adding any scalar to the sequential sum of their values. The absolute values of the factors in levels matter, therefore, only for the graphical visualization, while the analysis of the correlations between the factors and the series considers only the slope of the factor.
} 
Filtered 1 year yields

\begin{tabular}{rrrrrrrrrrr}
\hline & AT & BE & FI & FR & DE & GR & IR & IT & PT & SP \\
\hline 2011 & 0.34 & 0.22 & 0.28 & 0.41 & 0.28 & -0.60 & -0.12 & 0.12 & -0.28 & 0.15 \\
2012 & 0.44 & 0.50 & 0.12 & 0.34 & 0.19 & -0.56 & -0.05 & 0.62 & 0.30 & 0.55 \\
2016 & 0.32 & 0.34 & 0.39 & 0.33 & 0.36 & 0.13 & 0.29 & 0.14 & -0.01 & 0.17 \\
2017 & 0.67 & 0.31 & 0.69 & 0.32 & 0.59 & 0.01 & 0.62 & 0.10 & -0.18 & 0.02 \\
2018 & 0.33 & 0.12 & 0.45 & 0.21 & 0.54 & -0.46 & 0.28 & -0.78 & -0.12 & -0.25 \\
\hline
\end{tabular}

Table B.1: Yearly average Spearman correlation between the first factor and the 1 year yields filtered time series (first difference). Note: in the case of 1 year yields, many time series result being constant for long periods before 2010. In many case, therefore, it is not possible or meaningless to compute the Spearman correlation. We report the more complete results that we are able identify for this type of yields.

\begin{tabular}{rrrrrrrrrrr}
\hline & AT & BE & FI & FR & DE & GR & IR & IT & PT & SP \\
\hline 2011 & -0.04 & 0.05 & -0.08 & 0.02 & -0.02 & 0.33 & 0.06 & 0.08 & -0.03 & 0.10 \\
2012 & 0.20 & 0.26 & -0.16 & 0.15 & -0.05 & -0.55 & 0.04 & 0.70 & 0.16 & 0.68 \\
2016 & -0.03 & -0.09 & -0.06 & -0.05 & -0.07 & 0.05 & -0.07 & -0.20 & -0.13 & -0.21 \\
2017 & -0.10 & -0.03 & -0.09 & -0.02 & -0.12 & -0.18 & -0.12 & -0.02 & 0.07 & 0.04 \\
2018 & -0.02 & 0.01 & 0.01 & -0.06 & 0.10 & -0.33 & -0.15 & -0.55 & -0.06 & -0.26 \\
\hline
\end{tabular}

Table B.2: Yearly average Spearman correlation between the second factor and the 1 year yields filtered time series (first difference). Note: in the case of 1 year yields, many time series result being constant for long periods before 2010. In many case, therefore, it is not possible or meaningless to compute the Spearman correlation. We report the more complete results that we are able identify for this type of yields. 
Filtered 5 year yields

\begin{tabular}{rrrrrrrrrrrr}
\hline & AT & BE & FI & FR & DE & GR & IR & IT & NE & PT & SP \\
\hline 2003 & 0.99 & 0.98 & 0.00 & 0.98 & 0.97 & 0.00 & 0.88 & 0.98 & 0.97 & 0.98 & 0.98 \\
2004 & 0.66 & 0.66 & 0.00 & 0.64 & 0.64 & 0.00 & 0.65 & 0.65 & 0.66 & 0.66 & 0.66 \\
2005 & 0.66 & 0.66 & 0.00 & 0.64 & 0.65 & 0.00 & 0.52 & 0.64 & 0.65 & 0.66 & 0.64 \\
2006 & 0.98 & 1.00 & 0.00 & 0.99 & 1.00 & 0.00 & 0.39 & 0.98 & 0.98 & 1.00 & 1.00 \\
2007 & 0.99 & 0.99 & 0.37 & 1.00 & 0.99 & 0.98 & 0.78 & 0.98 & 0.99 & 0.99 & 1.00 \\
2008 & 0.94 & 0.96 & 0.96 & 0.96 & 0.93 & 0.85 & 0.92 & 0.89 & 0.97 & 0.94 & 0.96 \\
2009 & 0.86 & 0.85 & 0.84 & 0.86 & 0.81 & 0.47 & 0.62 & 0.72 & 0.88 & 0.73 & 0.81 \\
2010 & 0.81 & 0.54 & 0.84 & 0.84 & 0.84 & -0.20 & 0.09 & 0.19 & 0.84 & -0.08 & 0.12 \\
2011 & 0.66 & 0.28 & 0.59 & 0.64 & 0.53 & -0.18 & -0.20 & -0.08 & 0.61 & -0.24 & -0.04 \\
2012 & 0.81 & 0.81 & 0.34 & 0.78 & 0.13 & -0.03 & 0.06 & 0.59 & 0.57 & 0.07 & 0.58 \\
2013 & 0.92 & 0.89 & 0.92 & 0.92 & 0.86 & 0.07 & 0.48 & 0.73 & 0.92 & 0.41 & 0.59 \\
2014 & 0.44 & 0.45 & 0.38 & 0.43 & 0.32 & 0.44 & 0.48 & 0.52 & 0.41 & 0.49 & 0.51 \\
2015 & 0.90 & 0.92 & 0.86 & 0.92 & 0.79 & 0.04 & 0.84 & 0.74 & 0.88 & 0.62 & 0.71 \\
2017 & 0.89 & 0.92 & 0.89 & 0.90 & 0.88 & 0.01 & 0.90 & 0.61 & 0.89 & 0.43 & 0.60 \\
2018 & 0.90 & 0.85 & 0.92 & 0.87 & 0.93 & -0.32 & 0.82 & -0.40 & 0.89 & -0.17 & -0.04 \\
\hline
\end{tabular}

Table B.3: Yearly average Spearman correlation between the first factor and the 5 year yields filtered time series (first difference).

\begin{tabular}{rrrrrrrrrrrr}
\hline & AT & BE & FI & FR & DE & GR & IR & IT & NE & PT & SP \\
\hline 2006 & -0.07 & -0.08 & 0.00 & -0.07 & -0.08 & 0.00 & -0.01 & -0.05 & -0.04 & -0.08 & -0.09 \\
2007 & 0.04 & 0.05 & 0.01 & 0.05 & 0.04 & 0.08 & -0.12 & 0.09 & 0.05 & 0.06 & 0.05 \\
2008 & -0.12 & -0.05 & -0.12 & -0.12 & -0.22 & 0.35 & 0.05 & 0.20 & -0.09 & 0.02 & -0.06 \\
2009 & 0.00 & 0.11 & -0.07 & -0.06 & -0.14 & 0.46 & 0.18 & 0.26 & -0.07 & 0.25 & 0.19 \\
2010 & 0.14 & 0.52 & -0.00 & 0.06 & -0.08 & 0.74 & 0.20 & 0.70 & 0.01 & 0.78 & 0.75 \\
2011 & 0.07 & 0.37 & -0.07 & 0.10 & -0.18 & 0.48 & 0.34 & 0.42 & -0.08 & 0.41 & 0.42 \\
2012 & 0.21 & 0.38 & -0.48 & 0.24 & -0.58 & -0.06 & 0.24 & 0.86 & -0.25 & 0.20 & 0.84 \\
2013 & 0.05 & 0.09 & 0.05 & 0.05 & -0.07 & 0.33 & 0.44 & 0.64 & 0.04 & 0.83 & 0.72 \\
2014 & 0.07 & 0.08 & -0.01 & 0.07 & -0.10 & 0.83 & 0.44 & 0.64 & 0.03 & 0.74 & 0.64 \\
2015 & 0.19 & 0.21 & 0.15 & 0.20 & 0.00 & 0.50 & 0.34 & 0.74 & 0.17 & 0.79 & 0.75 \\
2017 & -0.04 & 0.04 & -0.07 & 0.11 & -0.05 & 0.06 & 0.09 & 0.54 & -0.07 & 0.57 & 0.54 \\
2018 & -0.01 & -0.03 & -0.01 & -0.03 & -0.01 & -0.11 & -0.03 & -0.12 & -0.01 & -0.13 & -0.11 \\
\hline
\end{tabular}

Table B.4: Yearly average Spearman correlation between the second factor and the 5 year yields filtered time series (first difference). 
Filtered 10 year yields

\begin{tabular}{rrrrrrrrrrrr}
\hline & AT & BE & FI & FR & DE & GR & IR & IT & NE & PT & SP \\
\hline 2003 & 0.96 & 0.99 & 0.99 & 0.98 & 0.99 & 0.00 & 0.99 & 0.99 & 0.98 & 0.98 & 0.98 \\
2004 & 0.82 & 0.82 & 0.83 & 0.82 & 0.82 & 0.00 & 0.83 & 0.82 & 0.82 & 0.79 & 0.82 \\
2005 & 0.94 & 0.99 & 0.99 & 0.99 & 0.99 & 0.00 & 0.99 & 0.98 & 0.92 & 0.74 & 0.98 \\
2006 & 0.95 & 1.00 & 0.99 & 0.99 & 1.00 & 0.00 & 0.94 & 0.98 & 0.99 & 0.78 & 0.99 \\
2007 & 0.40 & 0.40 & 0.40 & 0.40 & 0.40 & 0.20 & 0.20 & 0.40 & 0.40 & 0.40 & 0.40 \\
2008 & 0.95 & 0.98 & 0.95 & 0.96 & 0.91 & 0.85 & 0.92 & 0.90 & 0.96 & 0.94 & 0.95 \\
2009 & 0.86 & 0.86 & 0.85 & 0.86 & 0.79 & 0.50 & 0.61 & 0.75 & 0.87 & 0.72 & 0.80 \\
2010 & 0.73 & 0.57 & 0.79 & 0.78 & 0.77 & -0.07 & 0.01 & 0.32 & 0.77 & 0.09 & 0.21 \\
2011 & 0.51 & 0.16 & 0.52 & 0.48 & 0.50 & -0.12 & -0.16 & -0.11 & 0.51 & -0.09 & -0.11 \\
2012 & 0.75 & 0.64 & 0.83 & 0.71 & 0.87 & -0.70 & -0.14 & -0.46 & 0.83 & -0.54 & -0.42 \\
2013 & 0.42 & 0.43 & 0.41 & 0.43 & 0.37 & 0.16 & 0.28 & 0.32 & 0.41 & 0.18 & 0.29 \\
2014 & 0.69 & 0.71 & 0.67 & 0.71 & 0.59 & 0.29 & 0.68 & 0.63 & 0.67 & 0.50 & 0.63 \\
2015 & 0.93 & 0.96 & 0.90 & 0.95 & 0.87 & 0.03 & 0.90 & 0.78 & 0.92 & 0.54 & 0.73 \\
2016 & 0.92 & 0.94 & 0.91 & 0.94 & 0.81 & 0.01 & 0.92 & 0.84 & 0.90 & 0.66 & 0.79 \\
2017 & 0.74 & 0.77 & 0.75 & 0.78 & 0.71 & 0.06 & 0.78 & 0.60 & 0.74 & 0.48 & 0.59 \\
2018 & 0.97 & 0.94 & 0.98 & 0.96 & 0.96 & -0.14 & 0.93 & -0.06 & 0.97 & 0.18 & 0.29 \\
\hline
\end{tabular}

Table B.5: Yearly average Spearman correlation between the first factor and the 10 year yields filtered time series (first difference).

\begin{tabular}{rrrrrrrrrrrr}
\hline & AT & BE & FI & FR & DE & GR & IR & IT & NE & PT & SP \\
\hline 2006 & -0.04 & -0.08 & -0.08 & -0.08 & -0.08 & 0.00 & -0.09 & -0.08 & -0.07 & -0.18 & -0.09 \\
2007 & -0.06 & -0.03 & -0.05 & -0.06 & -0.08 & -0.17 & 0.08 & -0.00 & -0.05 & -0.01 & -0.04 \\
2008 & -0.15 & -0.10 & -0.19 & -0.15 & -0.26 & 0.20 & -0.04 & 0.13 & -0.13 & 0.03 & -0.05 \\
2009 & 0.13 & 0.12 & -0.03 & 0.00 & -0.15 & 0.64 & 0.49 & 0.36 & 0.02 & 0.40 & 0.24 \\
2010 & -0.11 & 0.01 & -0.10 & -0.09 & -0.09 & 0.09 & 0.14 & 0.11 & -0.12 & 0.10 & 0.12 \\
2011 & 0.12 & 0.63 & -0.17 & 0.17 & -0.28 & 0.61 & 0.45 & 0.72 & -0.17 & 0.52 & 0.70 \\
2012 & -0.06 & 0.09 & -0.19 & 0.01 & -0.30 & 0.58 & 0.37 & 0.77 & -0.17 & 0.75 & 0.79 \\
2013 & -0.01 & 0.02 & -0.04 & 0.01 & -0.07 & 0.50 & 0.31 & 0.37 & -0.04 & 0.43 & 0.41 \\
2014 & -0.02 & 0.04 & -0.04 & 0.03 & -0.17 & 0.88 & 0.44 & 0.64 & -0.06 & 0.74 & 0.62 \\
2015 & -0.00 & 0.05 & -0.06 & 0.03 & -0.11 & 0.93 & 0.22 & 0.42 & -0.03 & 0.59 & 0.44 \\
2016 & 0.03 & 0.12 & 0.02 & 0.13 & -0.09 & 0.27 & 0.27 & 0.46 & 0.01 & 0.54 & 0.45 \\
2017 & 0.02 & 0.12 & 0.01 & 0.16 & -0.03 & 0.20 & 0.17 & 0.55 & 0.01 & 0.64 & 0.56 \\
2018 & -0.01 & 0.05 & -0.01 & 0.04 & -0.05 & 0.28 & 0.05 & 0.39 & -0.03 & 0.36 & 0.33 \\
\hline
\end{tabular}

Table B.6: Yearly average Spearman correlation between the second factor and the 10 year yields filtered time series (first difference). 


\section{Appendix C. Additional Results}

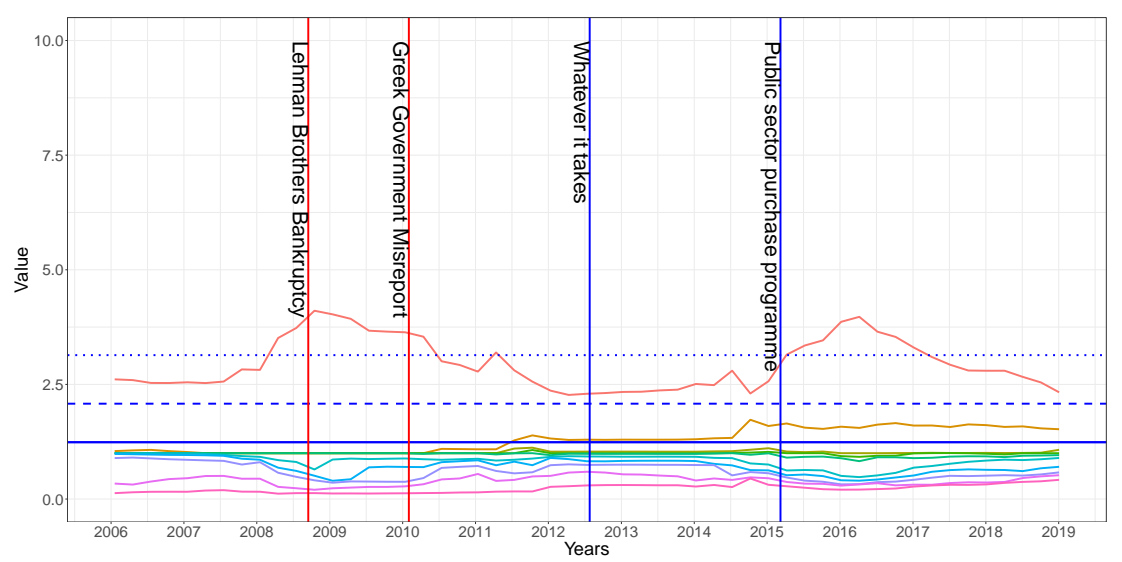

(a) One year yields

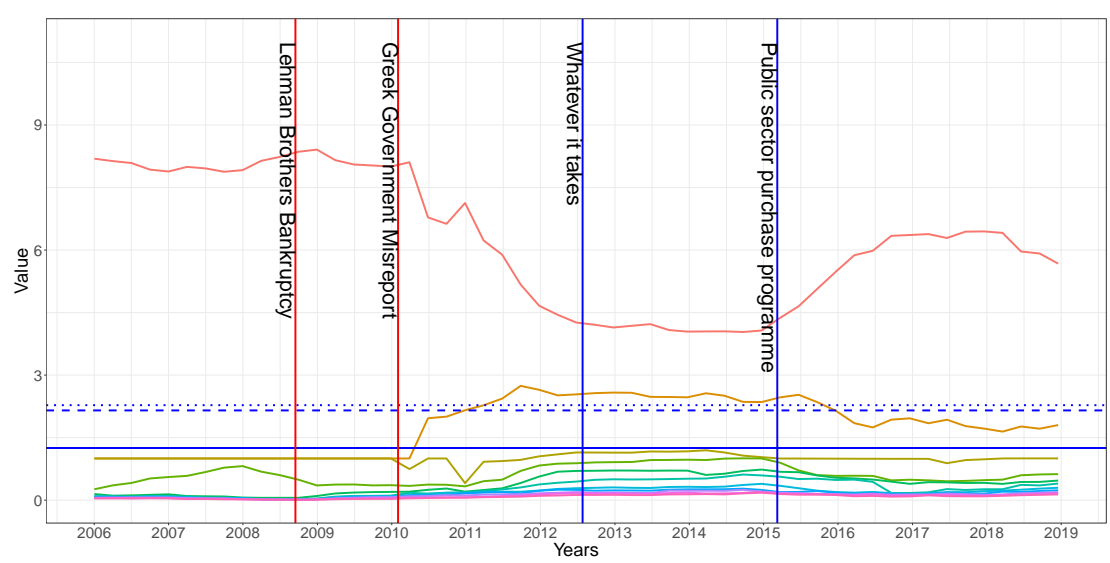

(b) Five year yields

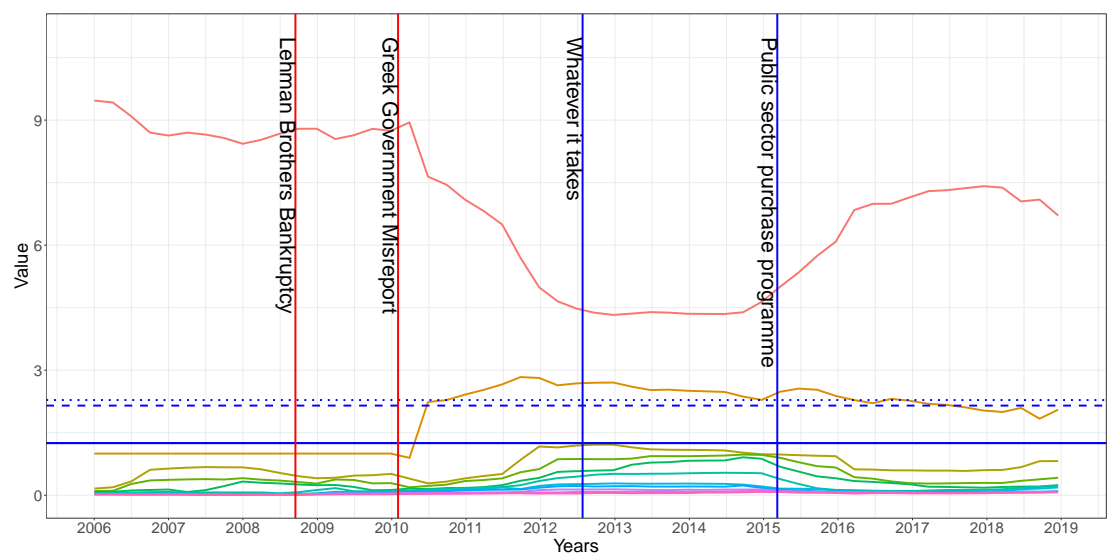

(c) Ten year yields

Figure C.1: Eigenvalue evolution with optimal parameters for first difference.

Notes: the full line indicates the Marčenko-Pastur theoretical bound, the dotted line indicates the simulated random model, and the thin dotted line indicates the rotational bound. For both the simulated random model and rotational random shuffling, 300 Monte Carlo simulations have been run. The dimension of the windows of the random matrix theory are reported in Table 2 in Section 6.2 (in short, windows' width of 783 observations, i.e. 3 years, and step of 65 observations, i.e. one quarter). 


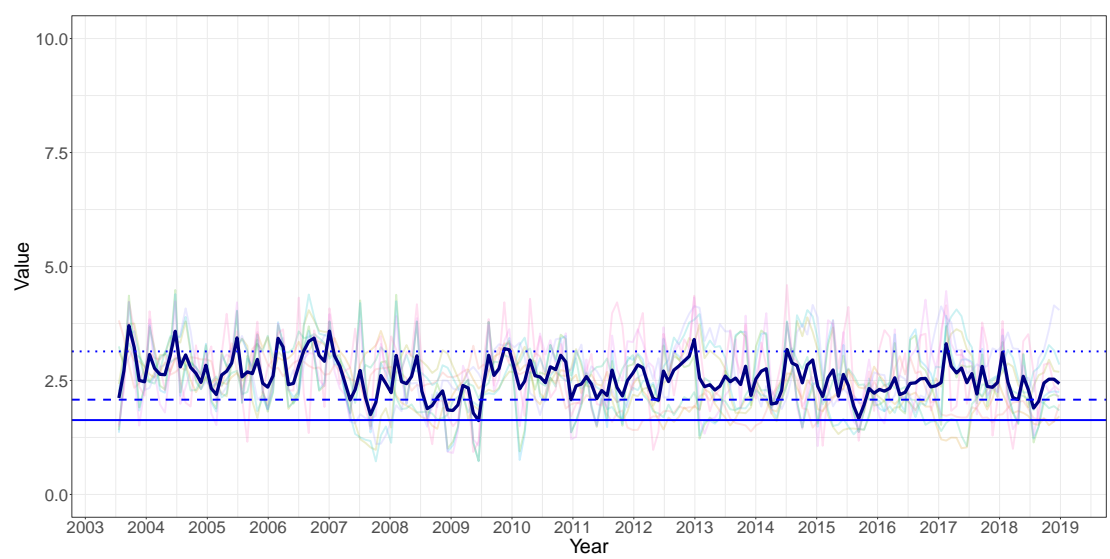

(a) One year yields

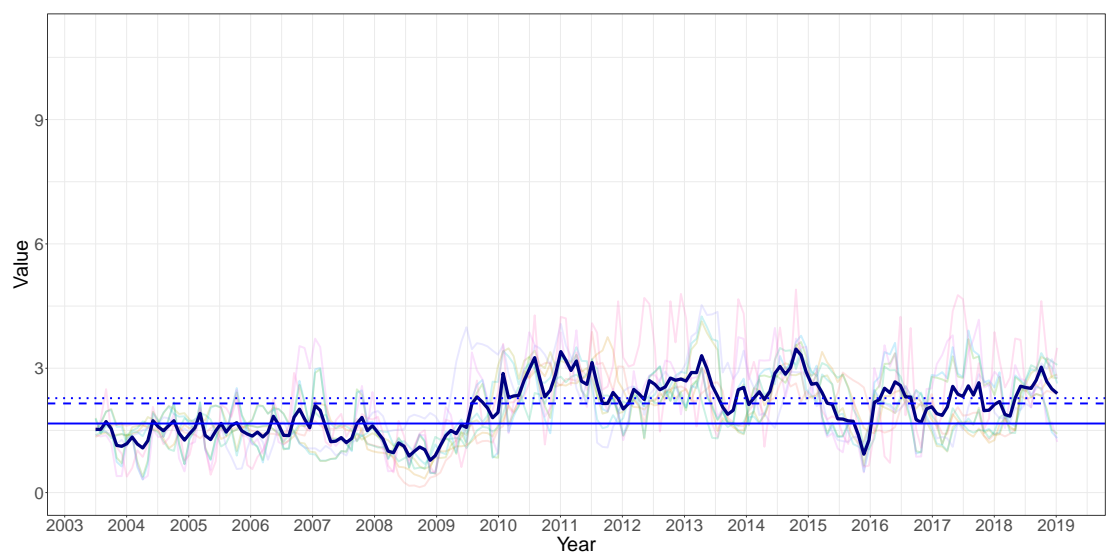

(b) Five year yields

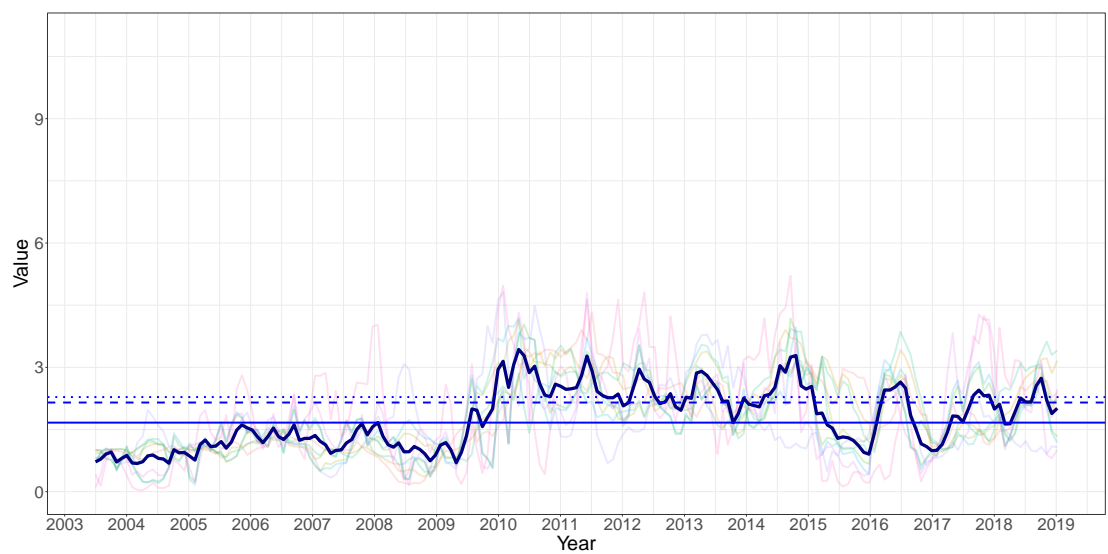

(c) Ten year yields

Figure C.2: Evolution of the second largest eigenvalue under different bandpass filters. The line in bold is the time average across the eigenvalues corresponding to the different frequency bands.

Notes: the full line indicates the Marčenko-Pastur theoretical bound, the dotted line indicates the simulated random model, and the thin dotted line indicates the rotational bound. For both the simulated random model and rotational random shuffling, 300 Monte Carlo simulations have been run. The full dark blue line indicates the mean of the values given by the different frequencies selection. The selection of the frequencies is discussed in Section 6.2. 